\title{
Discontinuous perturbations of nonhomogeneous strongly-singular Kirchhoff problems
}

\author{
Vicenţiu D. Rădulescu@e, Carlos Alberto Santos, Lais Santos and \\ Marcos L. M. Carvalho
}

\begin{abstract}
In this paper, we are concerned with a Kirchhoff problem in the presence of a strongly-singular term perturbed by a discontinuous nonlinearity of the Heaviside type in the setting of Orlicz-Sobolev space. The presence of both strongly-singular and non-continuous terms brings up difficulties in associating a differentiable functional to the problem with finite energy in the whole space $W_{0}^{1, \Phi}(\Omega)$. To overcome this obstacle, we establish an optimal condition for the existence of $W_{0}^{1, \Phi}(\Omega)$-solutions to a strongly-singular problem, which allows us to constrain the energy functional to a subset of $W_{0}^{1, \Phi}(\Omega)$ in order to apply techniques of convex analysis and generalized gradient in the sense of Clarke.
\end{abstract}

Mathematics Subject Classification. 35J25, 35J62, 35J75, 35J20, 35D30, 35B38.

Keywords. Non-local Kirchhoff problems, Strongly-singular nonlinearity, $\Phi$-Laplace operator, Discontinuous perturbation.

\section{Introduction}

In this paper, we are concerned in presenting equivalent conditions for the existence of three solutions for the quasilinear problem

$$
\left(Q_{\lambda, \mu}\right) \begin{cases}-M\left(\int_{\Omega} \Phi(|\nabla u|) d x\right) \Delta_{\Phi} u=\mu b(x) u^{-\delta}+\lambda f(x, u) & \text { in } \Omega, \\ u>0 & \text { in } \Omega, u=0 \text { on } \partial \Omega,\end{cases}
$$

which are linked to an optimal compatibility condition between $(b, \delta)$ for existence of solution to the strongly-singular problem

$$
(S) \begin{cases}-\Delta_{\Phi} u=b(x) u^{-\delta} & \text { in } \Omega \\ u>0 & \text { in } \Omega, u=0 \text { on } \partial \Omega\end{cases}
$$


with the boundary condition still in the sense of the trace.

Here, $M:[0, \infty) \rightarrow[0, \infty)$ is a continuous function, $f: \Omega \times(0, \infty) \rightarrow$ $(0, \infty)$ is of Heaviside type, $0<b \in L^{1}(\Omega), \delta>1, \lambda, \mu>0$ are real parameters. Moreover, $-\Delta_{\Phi} u=-\operatorname{div}(a(|\nabla u|) \nabla u)$ stands for the $\Phi$-Laplace operator, where $a:(0, \infty) \rightarrow(0, \infty)$ is a $C^{1}$-function that defines the increasing homeomorphism $\phi: \mathbb{R} \rightarrow \mathbb{R}$ given by

$$
\phi(t)= \begin{cases}a(|t|) t & \text { if } t \neq 0 \\ 0 & \text { if } t=0\end{cases}
$$

whose the associated N-function $\Phi: \mathbb{R} \rightarrow \mathbb{R}$ is given by $\Phi(t)=\int_{0}^{|t|} \phi(s) d s$.

The issue about existence of three solutions for a suitable range of parameters $\lambda, \mu>0$, for particular forms of Problem $\left(Q_{\lambda, \mu}\right)$, has been considered in the literature recently, principally in the context of non-singular problems $(\delta<0)$ and in the case in which $f$ is continuous, see for instance $[3,11,30,33]$ and references therein. There are few works for singular nonlinearities, we quote for example $[12,13,33]$ who considered $\Phi(t)=|t|^{p} / p, t>0,1<p<\infty$ and $M \equiv 1$ in $\left(Q_{\lambda, \mu}\right)$. We also refer to $[21,24,26]$ on recent developments to the study of local or nonlocal problems with nonstandard growth.

In [33], a singular problem for low dimensions was studied, while in [12] and [13] a singular problem for high dimensions was treated, but in both cases $f$ has been considered a Carathéodory function with suitable assumptions. More specifically, in [13], the singular perturbation was considered in the weak sense $(0<\delta<1)$, while in [12] they permitted $\delta>1$ by balancing the size of this $\delta$ with the existence of a $0<u \in C_{0}^{1}(\bar{\Omega})$ such that the product $b u^{-\delta}$ in $L^{\left(p^{*}\right)^{\prime}}(\Omega)$.

In this paper, we establish an optimal condition to the relationship between the power $\delta>1$ and the potential $b(x)>0$ to existence of three solutions to the singular problem $\left(Q_{\lambda, \mu}\right)$, independent of the dimension $N$, in the presence of both a discontinuous nonlinearity of the Heaviside type and a non-local term. More precisely, we prove how the existence of three solutions to $\left(Q_{\lambda, \mu}\right)$ is associated to the existence of solutions still in $W_{0}^{1, \Phi}(\Omega)$ to the problem $(S)$. Our approach is based on the existence of positive solution to the problem $(S)$, which provides a non-empty effective domain for the energy functional associated to $\left(Q_{\lambda, \mu}\right)$ and enable us to apply techniques of the generalized gradient in Clarke sense to get a multiplicity result.

Besides this, we prove qualitative results about these three solutions. We highlight how the non-local term $M$ should be to the discontinuity of the function $f$ be effectively attained by the solutions and how the level set of these solutions behaves exactly at the discontinuity point of $f$. To our knowledge, both the results of equivalent conditions and qualitative information on solutions are new in literature.

As our main results will be obtained via variational methods, we need to introduce the energy functional associated to Problem $\left(Q_{\lambda, \mu}\right)$. To do this, let us denote by $W_{0}^{1, \Phi}(\Omega)$ the Orlicz-Sobolev space associated to $\Phi$ and extend the function $f$ to $\mathbb{R}$ as $f(x, t)=0$ a.e in $\Omega$ and for all $t \leq 0$. From these, the functional naturally associated to $\left(Q_{\lambda, \mu}\right)$ is $I: W_{0}^{1, \Phi}(\Omega) \rightarrow \mathbb{R} \cup\{\infty\}$ defined 
by

$$
I(u)=\hat{M}\left(\int_{\Omega} \Phi(|\nabla u|) d x\right)-\lambda \int_{\Omega} F(x, u) d x+\mu \int_{\Omega} G(x, u) d x,
$$

where

$$
\hat{M}(t)=\int_{0}^{t} M(s) d s, F(x, t):=\int_{0}^{t} f(x, s) d s
$$

and $G: \Omega \times \mathbb{R} \rightarrow(-\infty, \infty]$ is defined by

$$
G(x, t)= \begin{cases}\frac{-b(x) t^{1-\delta}}{1-\delta} & \text { for } x \in \Omega \text { and } t>0 \\ +\infty & \text { for } x \in \Omega \text { and } t \leq 0\end{cases}
$$

To ease our future references, let us rewrite $I$ as $I=\Psi_{1}+\mu \Psi_{2}$, where

$$
\Psi_{1}(u)=\hat{M}\left(\int_{\Omega} \Phi(|\nabla u|) d x\right)-\lambda \int_{\Omega} F(x, u) d x
$$

and

$$
\Psi_{2}(u)=\int_{\Omega} G(x, u) d x .
$$

The main difficulty in treating strongly-singular problems consists in the fact that the energy functional associated to the equation neither belongs to $C^{1}$, in the sense of Fréchet differentiability, nor is defined in the whole space $W_{0}^{1, \Phi}(\Omega)$. In fact, when $\delta>1$ the functional $\Psi_{2}$ may not be proper, i.e. it may occur $\Psi_{2}(u)=\infty$, for all $u \in W_{0}^{1, \Phi}(\Omega)$.

Another difficulty exploited in this work is the presence of a more general quasilinear operator, which may be even nonhomogeneous. To deal with this situation, we approach the problem $\left(Q_{\lambda, \mu}\right)$ in Orlicz-Sobolev space setting. Below, let us state the assumptions about $\Phi$ that we will assume throughout this paper.

$\left(\phi_{0}\right): a \in C^{1}((0, \infty),(0, \infty))$ and $\phi$ is an increasing odd homeomorphisms from $\mathbb{R}$ onto $\mathbb{R}$;

$\left(\phi_{1}\right): 0<a_{-}:=\inf _{t>0} \frac{t \phi^{\prime}(t)}{\phi(t)} \leq \sup _{t>0} \frac{t \phi^{\prime}(t)}{\phi(t)}:=a_{+}<\infty$.

Let us denote by $\Phi_{*}$ the function whose inverse is given by $\left(\Phi_{*}\right)^{-1}(t)=$ $\int_{0}^{t} \Phi^{-1}(s) s^{-1-1 / N} d s, t>0$. In order to $\Phi_{*}$ be a N-function, we need to require

$$
\int_{0}^{1} \Phi^{-1}(s) s^{-1-1 / N} d s<\infty \text { and } \int_{1}^{\infty} \Phi^{-1}(s) s^{-1-1 / N} d s=\infty .
$$

In this case, $\Phi_{*}$ is a $\mathrm{N}$-function given by $\Phi_{*}(t)=\int_{0}^{|t|} \phi_{*}(s) d s$ for some increasing odd homeomorphisms $\phi_{*}: \mathbb{R} \rightarrow \mathbb{R}$. About $\Phi_{*}$, we will consider $\left(\phi_{2}\right): \phi_{+}<\phi_{-}^{*}:=\inf _{t>0} \frac{t \phi_{*}(t)}{\Phi_{*}(t)}$, where $1<\phi_{-}:=a_{-}+1 \leq a_{+}+1:=\phi_{+}$.

As another consequence of $\left(\phi_{0}\right)$ and $\left(\phi_{1}\right)$, the Orlicz space $L^{\Phi}(\Omega)$ coincides with the set (equivalence classes) of measurable functions $u: \Omega \rightarrow \mathbb{R}$ such 
that $\int_{\Omega} \Phi(|u|) d x<\infty$ and it is a Banach space endowed with the Luxemburg norm

$$
\|u\|_{\Phi}:=\inf \left\{\alpha>0: \int_{\Omega} \Phi\left(\frac{|u(x)|}{\alpha}\right) d x \leq 1\right\} .
$$

Associated to the space $L^{\Phi}(\Omega)$, we can set the Orlicz-Sobolev space $W^{1, \Phi}(\Omega)$ by

$$
W^{1, \Phi}(\Omega)=\left\{u \in L^{\Phi}(\Omega): u_{x_{i}} \in L^{\Phi}(\Omega), i=1, \cdots, N\right\}
$$

and deduce that it is a Banach space with respect to the norm

$$
\|u\|_{W^{1, \Phi}}=\|u\|_{\Phi}+\|\nabla u\|_{\Phi} .
$$

The Orlicz-Sobolev space $W_{0}^{1, \Phi}(\Omega)$ is naturally defined as the closure of $C_{0}^{\infty}(\Omega)$ in $W^{1, \Phi}(\Omega)$-norm, under the hypothesis $\left(\phi_{1}\right)$. For more information about the Orlicz and Orlicz-Sobolev spaces, we refer $[1,17,18]$.

About $M$, let us assume

$(M): M(t) \geq m_{0} t^{\alpha-1}$ for all $t \geq 0$ and for some $\alpha>0$ such that $\Phi_{\alpha} \prec \prec \Phi_{*}$, that is, $\lim _{t \rightarrow \infty} \frac{\Phi_{\alpha}(\tau t)}{\Phi_{*}(t)}=0$ for all $\tau>0$, where $\Phi_{\alpha}(t):=\Phi\left(t^{\alpha}\right)$.

To conclude our assumptions, let us suppose that $f: \Omega \times(0, \infty) \longrightarrow \mathbb{R}^{+}$ is a measurable function such that $f(x, t)=0$ a.e. in $\Omega \times(-\infty, 0]$ and

$\left(f_{0}\right): f(x, \cdot) \in \mathbf{C}(\mathbb{R}-\{\tilde{a}\})$ for some $\tilde{a}>0,-\infty<f(x, \tilde{a}-0)<f(x, \tilde{a}+0)<$ $\infty, x \in \Omega$, where

$$
f(x, \tilde{a}-0):=\lim _{s \rightarrow \tilde{a}^{-}} f(x, s), f(x, \tilde{a}+0):=\lim _{s \rightarrow \tilde{a}^{+}} f(x, s),
$$

$\left(f_{1}\right)$ : there exists an odd increasing homeomorphism $h$ from $\mathbb{R}$ onto $\mathbb{R}$ and nonnegative constants $a_{1}, a_{2}$ and $a_{3}$ such that

$|\eta| \leq a_{1}+a_{2} \widetilde{H}^{-1} \circ H\left(a_{3}|t|\right)$ for all $\eta \in \partial F(x, t), t \in \mathbb{R}$ and $x \in \bar{\Omega}$,

where $H(t)=\int_{0}^{|t|} h(s) d s$ is a N-function satisfying $\Delta_{2}(\tilde{H}$ is the its complementary function) such that $H \prec \prec \Phi_{*}$ and

$$
\frac{t h(t)}{H(t)} \leq h_{+} \text {for all } t \geq t_{0} \text { with } 1<h_{+} \leq \frac{\phi_{-}^{*}}{2}+1,
$$

for some $t_{0}>0$,

$\left(f_{2}\right): \lim _{t \rightarrow 0^{+}} \frac{\sup _{\bar{\Omega}} F(x, t)}{t^{\alpha \phi}+}=0$,

$\left(f_{3}\right): \lim _{t \rightarrow \infty} \frac{\sup _{\bar{\Omega}} F(x, t)}{t^{\alpha \phi_{-}}}=0$.

Before stating the main results, let us clarify what we mean by a solution of $\left(Q_{\lambda, \mu}\right)$.

Definition 1.1. A function $u \in W_{0}^{1, \Phi}(\Omega)$ is a solution to the problem $\left(Q_{\lambda, \mu}\right)$ if $u>0$ a.e in $\Omega, b u^{-\delta} \varphi \in L^{1}(\Omega)$ and

$$
\begin{aligned}
M & \left(\int_{\Omega} \Phi(|\nabla u|) d x\right) \int_{\Omega} a(|\nabla u|) \nabla u \nabla \varphi d x \\
& =\int_{\Omega}\left[\mu \frac{b(x)}{u^{\delta}}+\lambda f(x, u)\right] \varphi d x \text { for all } \varphi \in W_{0}^{1, \Phi}(\Omega) .
\end{aligned}
$$


Under the hypothesis $\left(f_{0}\right)$, a solution $0<u \in W_{0}^{1, \Phi}(\Omega)$ of the problem $\left(Q_{\lambda, \mu}\right)$ has to satisfy

$$
\begin{aligned}
& \int_{\Omega}(f(x, u(x)-0)-f(x, u(x)+0)) u \chi_{\{x \in \Omega: u(x)=\tilde{a}\}}(x) \varphi d x \\
& \quad=0 \text { for all } \varphi \in W_{0}^{1, \Phi}(\Omega),
\end{aligned}
$$

where $\chi_{\{x \in \Omega: u(x)=\tilde{a}\}}$ stands for the characteristic function of the set $\{x \in$ $\Omega: u(x)=\tilde{a}\}$. Next, we state that (4) is satisfied, under additional assumptions on $f$ and $b$, by showing that meas $\{x \in \Omega: u(x)=\tilde{a}\}=0$, where meas stands for the Lebesgue measure.

Theorem 1.1. Assume $f$ satisfies $\left(f_{0}\right),\left(f_{1}\right)$ and $0<b \in L^{1}(\Omega)$ holds. If $u \in$ $W_{0}^{1, \Phi}(\Omega)$ is such that:

(i) $u$ is either a local minimum or a local maximum of $I$, then meas $\{x \in$ $\Omega: u(x)=\tilde{a}\}=0$,

(ii) $u$ is a critical point of $I$ and $b \in L_{\mathrm{loc}}^{2}(\Omega)$, then meas $\{x \in \Omega:|\nabla u(x)|=$ $0\}=0$. In particular, meas $\{x \in \Omega: u(x)=c\}=0$ for each $c>0$.

Moreover, if u satisfies $(i)$ or $($ ii) above, then:

(iii) $u$ is a solution of Problem $\left(Q_{\lambda, \mu}\right)$,

(iv) there exists $C>0$ such that $u(x) \geq C d(x)$ for $x \in \bar{\Omega}$, where $d$ stands for the distance function to the boundary $\partial \Omega$,

(v) u solves $\left(Q_{\lambda, \mu}\right)$ almost everywhere in $\Omega$ if in addition $b d^{-\delta} \in L^{\tilde{H}}(\Omega)$.

Our main result on the multiplicity of solutions can be stated as follows.

Theorem 1.2. Assume $\delta>1, b \in L^{1}(\Omega) \cap L_{\text {loc }}^{2}(\Omega),\left(\phi_{0}\right)-\left(\phi_{2}\right),\left(f_{0}\right)-\left(f_{3}\right)$ and $(M)$ hold. Then, the below claims are equivalents:

(i) there exists $0<u_{0} \in W_{0}^{1, \Phi}(\Omega)$ such that $\int_{\Omega} b u_{0}^{1-\delta} d x<\infty$,

(ii) the problem $(S)$ admits a (unique) weak solution $u \in W_{0}^{1, \Phi}(\Omega)$ such that $u(x) \geq C d(x)$ for $x \in \bar{\Omega}$ for some $C>0$ independent of $u$,

(iii) for each $\lambda>\lambda^{*}$, there exists $\mu_{\lambda}>0$ such that for $\mu \in\left(0, \mu_{\lambda}\right]$, the problem $\left(Q_{\lambda, \mu}\right)$ admits at least three solutions, being two local minima and the other one a mountain pass critical point of the functional I, where

$$
\lambda^{*}=\inf \left\{\frac{\hat{M}\left(\int_{\Omega} \Phi(|\nabla u|)\right)}{\int_{\Omega} F(x, u) d x}: u \in W_{0}^{1, \Phi}(\Omega) \text { and } \int_{\Omega} F(x, u) d x>0\right\} \text {. }
$$

Moreover, for each of such solutions the meas $\{x \in \Omega: u(x)=\tilde{a}\}=0$. Besides this, $u$ solves $\left(Q_{\lambda, \mu}\right)$ almost everywhere in $\Omega$ if in addition $b d^{-\delta} \in L^{\tilde{H}}(\Omega)$ and

(iv) either $M$ is non-decreasing and $f(x, t)=f(x)$ for all $0<t<1$ and a.e. $x \in \Omega$,

(v) or $M$ is such that a Comparison Principle holds to Problem $\left(Q_{0, \mu}\right)$ and $\alpha \phi_{-}>1$,

then there exists $\tilde{a}^{\star}>0$ such that

$$
\text { meas }\left\{x \in \Omega: u(x)>\tilde{a} \text { and } u \text { is a solution of }\left(Q_{\lambda, \mu}\right)\right\}>0
$$


for each $0<\tilde{a}<\tilde{a}^{\star}$ given .

Remark 1.1. About the above theorem, we still highlight the following facts:

(i) the equivalency between $(i)$ and (ii) holds true without assuming $b \in$ $L_{\mathrm{loc}}^{2}(\Omega)$,

(ii) each one of such solutions given by (iii) is such that $u(x) \geq C d(x)$ for $x \in \bar{\Omega}$, for some $C>0$ dependent on $u$,

(iii) in particular, Theorem 1.2 shows that a perturbation by a Heaviside function is enough to break the uniqueness of solution of the pure singular problem $(S)$ to produce at least three ones for the problem

$$
\begin{cases}-\Delta_{\Phi} u=\mu b(x) u^{-\delta}+\lambda H(u-a) & \text { in } \Omega, \\ u>0 & \text { in } \Omega, u=0 \text { on } \partial \Omega,\end{cases}
$$

for appropriate parameters $\lambda, \mu>0$, where $H$ stands for the Heaviside function. This is new to literature of singular elliptic problems even to Laplacian operator.

In [19], Lazer and McKenna has proven that problem $(S)$ admits solution still in $H_{0}^{1}(\Omega)$ if, and only if, $\delta<3$ when $0<b_{0} \leq b \in L^{\infty}(\Omega)$ and $\Phi(t)=|t|^{2} / 2$ in $(S)$. Mohammed, in [25], considered $\Phi(t)=|t|^{p} / p(p>1)$ in $(S)$ and proved that the sharp power in this case is given by $(2 p-1) /(p-1)$. As a consequence of Theorem 1.2, we are able to find a $\delta_{q}>1$ such that the problem $(S)$ still admits a solution in $W_{0}^{1, \Phi}(\Omega)$ for all $\delta<\delta_{q}$, where $\delta_{q}$ depends on the summability $L^{q}(\Omega)$ of $b$. This is the content of the next corollary.

Corollary 1.1. Assume $\left(\phi_{0}\right),\left(\phi_{1}\right)$ and $\left(\phi_{2}\right)$ hold. If $0<b \in L^{q}(\Omega)$ for some $q>1$ and

$$
1<\delta<\frac{q\left(2 \phi_{+}-1\right)-\phi_{+}}{q\left(\phi_{+}-1\right)}:=\delta_{q},
$$

then the problem $(S)$ admits (unique) weak solution.

Although no answer about $\delta_{q}>1$ be the sharp power for the existence of solution still in $W_{0}^{1, \Phi}(\Omega)$ has been provided, we observe that $\delta_{q} \rightarrow$ $\left(2 \phi_{+}-1\right) /\left(\phi_{+}-1\right)$ as $q \rightarrow \infty$ and this limit is the sharp value obtained both by $[19,25]$ for the cases $\Phi(t)=|t|^{2} / 2$ and $\Phi(t)=|t|^{p} / p(p>1)$, respectively.

In particular, as a consequence of Theorem 1.2 and Corollary 1.1, we have the following.

Corollary 1.2. Assume $\left(\phi_{0}\right),\left(\phi_{1}\right),\left(\phi_{2}\right),(M)$ and $\left(f_{0}\right)-\left(f_{3}\right)$ hold. If $b \in L^{q}(\Omega)$ for some $q>1$ and $1<\delta<\delta_{q}$, then for each $\lambda>\lambda^{*}$ given, there exists $\mu_{\lambda}>0$ such that for $\mu \in\left(0, \mu_{\lambda}\right]$ the problem $\left(Q_{\lambda, \mu}\right)$ admits at least three weak solutions with the same properties as those found in item-iii) in Theorem 1.2.

It is worth mentioning that the above theorems improve or complement the related results in the literature both by the presence of the Kirchhoff term, the summability assumption on the potential $b$, the strongly-singular term and the non-homogeneity of the operator. Our results contribute to the literature principally by: 
(i) Theorem 1.1 unify some results on $\Delta_{p}$-Laplacian operator, with $1<p<$ $\infty$, to $\Phi$-Laplacian operator, see for instance $[2,22]$.

(ii) Theorem 1.2 establishes necessary and sufficient conditions for existence of multiple solutions for the problem $\left(Q_{\lambda, \mu}\right)$, by connecting and extending the principal result in Yijing [31] to a non-homogeneous operator;

(iii) Theorem 1.2 extends the principal result in Faraci et al. [12] and complements the main result in [13], principally by considering a non-homogeneous operator, an optimal condition on the pair $(b, \delta)$ to existence of three solutions, a discontinuity of the Heaviside type and including a Kirchhoff term;

(iv) Corollary 1.1 gives us an explicit range of variation of $\delta$, in which the existence of solution in $W_{0}^{1, \Phi}(\Omega)$ for $(S)$ is still guaranteed. In particular, when $\Phi(t)=|t|^{p} / p$ and $b_{0} \leq b(x) \in L^{\infty}(\Omega)$ for some constant $b_{0}>0$, the value $\delta_{q}$ coincides with the sharp values obtained in $[16,19]$;

(v) Corollary 1.2 complements the principal result in [12] by showing an explicit variation to $\delta$, where the multiplicity is still ensured, namely,

$$
0<\delta<\frac{p(N-1)}{N(p-1)}=\delta_{\left(p^{*}\right)^{\prime}},
$$

To ease the reading, from now on let us assume the assumptions $\left(\phi_{0}\right)$, $\left(\phi_{1}\right),\left(\phi_{2}\right),(M)$ and gather below some functional that appear throughout the paper.

- $\hat{M}(t)=\int_{0}^{t} M(s) d s, t \in \mathbb{R}$,

- $\Psi_{1}(u)=\hat{M}\left(\int_{\Omega} \Phi(|\nabla u|) d x\right)-\lambda \int_{\Omega} F(x, u) d x$,

- $\Psi_{2}(u)=\int_{\Omega} G(x, u) d x$,

- $\mathcal{P}(u)=\int_{\Omega} \Phi(|\nabla u|) d x$,

- $J(u)=\int_{\Omega} \Phi(|\nabla u|) d x+\frac{1}{\delta-1} \int_{\Omega} b(x)|u|^{1-\delta} d x$,

- $J_{1}(u):=(\hat{M} \circ \mathcal{P})(u)=\hat{M}\left(\int_{\Omega} \Phi(|\nabla u|) d x\right)$,

- $J_{2}(u)=\int_{\Omega} F(x, u) d x$,

- $I=\Psi_{1}+\mu \Psi_{2}=J_{1}-\lambda J_{2}+\mu \Psi_{2}$,

- $-(M \circ \mathcal{P})(\cdot) \Delta_{\Phi}(\cdot): W_{0}^{1, \Phi}(\Omega) \rightarrow\left(W_{0}^{1, \Phi}(\Omega)\right)^{\prime}$ is understood as

$\left\langle-(M \circ \mathcal{P})(u) \Delta_{\Phi} u, \varphi\right\rangle:=(M \circ \mathcal{P})(u) \int_{\Omega} a(|\nabla u|) \nabla u \nabla \varphi d x, \forall \varphi \in W_{0}^{1, \Phi}(\Omega)$.

This paper is organized as follows. In Sect. 2, we present some preliminary knowledge on the Orlicz-Sobolev spaces and some results of non-smooth analysis related to our problem. The Sect. 3 is reserved to prove Theorem 1.1, while in Sect. 4 we prove Theorem 1.2.

\section{Non-smooth analysis for locally Lipschitz functional}

In this section, we are going to remember some facts related to non-smooth analysis. However, one of the principal contribution of this section is establishing appropriated assumptions under the $\mathrm{N}$-function $\Phi$, the non-local term $M$ 
and the discontinuous function $f$ that make possible to approach $(i i \Longrightarrow i i i)$, in Theorem 1.2, via Ricceri's multiplicity theorem [28].

Under our hypotheses and the decomposition of the functional $I$ into $\Psi_{1}$ plus $\Psi_{2}$, that is,

$$
I=\Psi_{1}+\mu \Psi_{2},
$$

we have written $I$ as a sum of a locally Lipschitz functional $\Psi_{1}$ and a convex one $\Psi_{2}$ (see (1) and (2)). Below, let us recall few notations and results on the Critical Point Theory for the functional $\Psi_{1}$ and $\Psi_{2}$. We refer the reader to Carl, Le \& Motreanu [4], Chang [5], Clarke [8] and references therein for more details about this issue.

Let us begin by remembering that the generalized directional derivative of $\Psi_{1}$ at $u \in W_{0}^{1, \Phi}(\Omega)$ in the direction of $v \in W_{0}^{1, \Phi}(\Omega)$ is defined by

$$
\Psi_{1}^{0}(u ; v)=\limsup _{h \rightarrow 0} \frac{\Psi_{1}(u+h+\lambda v)-\Psi_{1}(u+h)}{\lambda}
$$

and the subdifferential of $\Psi_{1}^{0}(u ; \cdot)$ at $z \in W_{0}^{1, \Phi}(\Omega)$ is given by

$$
\begin{gathered}
\partial \Psi_{1}^{0}(u ; z)=\left\{\mu \in\left(W_{0}^{1, \Phi}(\Omega)\right)^{\prime}: \Psi_{1}^{0}(u ; v) \geq \Psi_{1}^{0}(u ; z)\right. \\
\left.+\langle\mu, v-z\rangle \text { for all } v \in W_{0}^{1, \Phi}(\Omega)\right\},
\end{gathered}
$$

since $\Psi_{1}^{0}(u ; \cdot)$ is a convex function. In particular, $\partial \Psi_{1}^{0}(u ; 0)$ is named by the generalized gradient of $\Psi_{1}$ at $u$ and denoted by $\partial \Psi_{1}(u)$.

About the functional $\Psi_{2}$, its effective domain is defined by $\operatorname{Dom}\left(\Psi_{2}\right)=$ $\left\{u \in W_{0}^{1, \Phi}(\Omega): \Psi_{2}(u)<\infty\right\}$ and a point $u \in \operatorname{Dom}\left(\Psi_{2}\right)$ is called a critical point of the functional $I$ if

$$
\Psi_{1}^{0}(u ; v-u)+\Psi_{2}(v)-\Psi_{2}(u) \geq 0, \forall v \in W_{0}^{1, \Phi}(\Omega) .
$$

In this context, we say that $I$ satisfies the Palais-Smale condition (the condition (PS) for short) if:

$$
\begin{gathered}
\text { " }\left\{u_{n}\right\} \subset W_{0}^{1, \Phi}(\Omega) \text { is such that } I\left(u_{n}\right) \rightarrow c \text { and } \\
\Psi_{1}^{0}\left(u_{n} ; v-u_{n}\right)+\Psi_{2}(v)-\Psi_{2}\left(u_{n}\right) \geq-\epsilon_{n}\left\|v-u_{n}\right\|, \quad \forall v \in W_{0}^{1, \Phi}(\Omega),
\end{gathered}
$$

where $\epsilon_{n} \rightarrow 0^{+}$, then $\left\{u_{n}\right\}$ possesses a convergent subsequence."

In order to prove the next result, let us define the functionals

$$
J_{1}(u):=\hat{M}(\mathcal{P}(u)) \text { and } J_{2}(u):=\int_{\Omega} F(x, u) d x,
$$

where $\mathcal{P}$ is defined by

$$
\mathcal{P}(u)=\int_{\Omega} \Phi(|\nabla u|) d x .
$$

It is well know that, under the hypotheses $\left(\phi_{0}\right)$ and $\left(\phi_{1}\right)$, the functional $\mathcal{P}$ is sequentially weakly lower semicontinuous and $C^{1}$ with

$$
\left\langle\mathcal{P}^{\prime}(u), \varphi\right\rangle=\int_{\Omega} a(|\nabla u|) \nabla u \nabla \varphi d x, \forall \varphi \in W_{0}^{1, \Phi}(\Omega) .
$$


Moreover, $\mathcal{P}^{\prime}: W_{0}^{1, \Phi}(\Omega) \rightarrow W_{0}^{-1, \tilde{\Phi}}(\Omega)$ is a strictly monotonic operator of the type $\left(S_{+}\right)$. Thus, we can rewrite $I$ as

$$
I=\Psi_{1}+\mu \Psi_{2}=J_{1}-\lambda J_{2}+\mu \Psi_{2},
$$

where $J_{1}$ is $C^{1}, J_{2}$ is locally Lipschitz and $\Psi_{2}$ is a convex functional.

Lemma 2.1. Suppose $\left(\phi_{0}\right),\left(\phi_{1}\right),\left(f_{0}\right)$ and $\left(f_{1}\right)$ holds. Then,

(i) $\left.J_{1} \in C^{1}\left(W_{0}^{1, \Phi}(\Omega), \mathbb{R}\right)\right)$ and

$$
\left\langle J_{1}^{\prime}(u), \varphi\right\rangle=M(\mathcal{P}(u)) \int_{\Omega} a(|\nabla u|) \nabla u \nabla \varphi d x, \forall \varphi \in W_{0}^{1, \Phi}(\Omega),
$$

(ii) $J_{2} \in \operatorname{Lip}_{\text {loc }}\left(W_{0}^{1, \Phi}(\Omega), \mathbb{R}\right)$ and

$$
\partial J_{2}(u) \subseteq\left\{w \in\left(L^{H}(\Omega)\right)^{\prime}: w(x) \in \partial F(x, u(x)) \text { a.e. } x \in \Omega\right\} .
$$

In particular, for each $w \in \partial J_{2}(u)$, there exists a unique $\omega \in L^{\tilde{H}}(\Omega)$ such that

$$
\begin{gathered}
\omega \in[f(x, u(x)-0), f(x, u(x)+0)] \quad \text { a.e. } x \in \Omega \text { and } \\
\langle w, \varphi\rangle=\int_{\Omega} \omega \varphi d x, \forall \varphi \in W_{0}^{1, \Phi}(\Omega),
\end{gathered}
$$

(iii) $J_{1}^{\prime}$ is of type $\left(S_{+}\right)$, that is,

"if $u_{n} \rightarrow u$ and $\lim _{n \rightarrow \infty} \sup \left\langle J_{1}^{\prime}\left(u_{n}\right), u_{n}-u\right\rangle \leq 0$, then $u_{n} \rightarrow u$ in $W_{0}^{1, \Phi}(\Omega)$ ".

$(i v)$ if $u_{n} \rightarrow u$ in $W_{0}^{1, \Phi}(\Omega)$, then $J_{2}^{0}\left(u_{n} ; u_{n}-u\right) \rightarrow 0$ and,

$$
\left\langle\eta_{n}, u_{n}-u\right\rangle=\int_{\Omega} \eta_{n}\left(u_{n}-u\right) d x \rightarrow 0, \forall \eta_{n} \in \partial J_{2}\left(u_{n}\right),
$$

$(v)$ if $u_{n} \rightarrow u$ in $W_{0}^{1, \Phi}(\Omega)$, then $J_{2}\left(u_{n}\right) \rightarrow J_{2}(u)$,

(vi) $J_{1}$ is sequentially weakly lower semicontinuous in $W_{0}^{1, \Phi}(\Omega)$,

(vii) $\Psi_{1} \in \operatorname{Lip}_{\text {loc }}\left(W_{0}^{1, \Phi}(\Omega) ; \mathbb{R}\right)$ is sequentially weakly lower semicontinuous and $\Psi_{1}^{0}$ is of the type $\left(S_{+}\right)$.

Proof. First, we note that the item $(i)$ is an immediate consequence of assumptions on $M$ and properties of $\mathcal{P}$. Next, we present a summary proof of the other items.

(ii) Let $\widetilde{J}_{2}: L^{H}(\Omega) \rightarrow \mathbb{R}$ be a functional defined by $\widetilde{J}_{2}(u)=\int_{\Omega} F(x, u) d x, u \in$ $L^{H}(\Omega)$. So, it follows from Theorem 1.1 in $[20]$ that $\widetilde{J}_{2} \in \operatorname{Lip}_{\text {loc }}\left(L^{H}(\Omega) ; \mathbb{R}\right)$ and

$$
\partial \widetilde{J}_{2}(u) \subseteq\left\{w \in\left(L^{H}(\Omega)\right)^{\prime}: w(x) \in \partial F(x, u(x)) \text { a.e. } x \in \Omega\right\} .
$$

Since ${\overline{W_{0}^{1, \Phi}(\Omega)}}^{L^{H}}=L^{H}(\Omega)$, we are able to apply [5, Theorem 2.2] to conclude that $J_{2}=\left.\widetilde{J}_{2}\right|_{W_{0}^{1, \Phi}(\Omega)}$ is locally Lipschitz continuous and

$$
\partial J_{2}(u) \subseteq \partial \widetilde{J}_{2}(u) \subseteq\left\{w \in\left(L^{H}(\Omega)\right)^{\prime}: w(x) \in \partial F(x, u(x)) \text { a.e. } x \in \Omega\right\} .
$$

The conclusion of the proof is a direct consequence of Theorem 1.1 in [20] and classical Riesz Theorem for Orlicz spaces, see for instance [27]. 
(iii) This conclusion is a consequence of item $(i)$ and the fact that $\mathcal{P}^{\prime}$ is of the type $\left(S_{+}\right)$.

(iv) Let $u_{n} \rightarrow u$ and $\eta_{n} \in \partial J_{2}\left(u_{n}\right)$. Since $\eta_{n} \in\left(L^{H}(\Omega)\right)^{\prime}$, the Riesz theorem for Orlicz spaces implies that there exists a unique $\eta_{n} \in L^{\widetilde{H}}(\Omega)$, still denoted by $\eta_{n}$, such that

$$
\left\langle\eta_{n}, u_{n}-u\right\rangle=\int_{\Omega} \eta_{n}\left(u_{n}-u\right) d x .
$$

Besides this, by using $\left(f_{1}\right), H \in \Delta_{2}$ and Young's inequality, we obtain

$$
\begin{aligned}
\left|\eta_{n}\left(u_{n}-u\right)\right| & \leq a_{1}\left|u_{n}-u\right|+a_{2} \widetilde{H}^{-1} \circ H\left(a_{3}\left|u_{n}-u\right|+a_{3}|u|\right)\left|u_{n}-u\right| \\
& \leq C\left(\left|u_{n}-u\right|+H\left(\left|u_{n}-u\right|+|u|\right)\right),
\end{aligned}
$$

which leads us to conclude that $\left|\eta_{n}\left(u_{n}-u\right)\right| \leq g(x)$ for some $g \in L^{1}(\Omega)$, after using the compact embedding $W_{0}^{1, \Phi}(\Omega) \hookrightarrow L^{H}(\Omega)$ and Lemma 5.3 in [14]. As $u_{n} \rightarrow u$ a.e in $\Omega$, the first claim follows by Lebesgue's theorem.

To end the proof, it follows from Proposition 2.171 in [4] that there exists $\widetilde{\eta}_{n} \in \partial J_{2}\left(u_{n}\right)$ such that $J_{2}^{0}\left(u_{n} ; v\right)=\left\langle\widetilde{\eta}_{n}, v\right\rangle$, for all $v \in W_{0}^{1, \Phi}(\Omega)$. Hence, we obtain from above conclusion that $J_{2}^{0}\left(u_{n} ; u_{n}-u\right)=\left\langle\widetilde{\eta}_{n}, u_{n}-\right.$ $u\rangle \rightarrow 0$.

$(v)$ As in the previous item, by using $\left(f_{1}\right)$ and dominated convergence the result follows.

(vi) This item is a consequence of the continuity and monotonicity of $\hat{M}$ and the fact that $\mathcal{P}$ is sequentially weakly lower semicontinuous in $W_{0}^{1, \Phi}(\Omega)$.

(vii) By items $(i)$ and (ii) above, we have $\Psi_{1} \in \operatorname{Lip}_{\text {loc }}\left(W_{0}^{1, \Phi}(\Omega) ; \mathbb{R}\right)$. Besides this, we get from item $(i v)$ and $\left(f_{1}\right)$ that $\Psi_{1}$ is sequentially weakly lower semicontinuous. Let $u_{n} \rightarrow u$ such that $\limsup _{n \rightarrow \infty} \Psi_{1}^{0}\left(u_{n} ; u_{n}-u\right) \leq 0$. Then, (iii) and (iv) above lead us to

$$
\begin{aligned}
& \limsup _{n \rightarrow \infty}\left\langle-(M \circ \mathcal{P})\left(u_{n}\right) \Delta_{\Phi} u_{n}, u_{n}-u\right\rangle \\
& =\limsup _{n \rightarrow \infty}\left\langle-(M \circ \mathcal{P})\left(u_{n}\right) \Delta_{\Phi} u_{n}, u_{n}-u\right\rangle \\
& \quad-\lambda \lim _{n \rightarrow \infty} J_{2}^{0}\left(u_{n} ; u_{n}-u\right)=\limsup _{n \rightarrow \infty} \Psi_{1}^{0}\left(u_{n} ; u_{n}-u\right) \leq 0,
\end{aligned}
$$

which implies the claimed, after using the $($ iii $)$. This ends the proof.

The next lemma gives us some properties regarding $\Psi_{2}$.

Lemma 2.2. Assume $0<b \in L^{1}(\Omega)$. If Problem $(S)$ admits a solution in $W_{0}^{1, \Phi}(\Omega)$, then $\Psi_{2}$ is a proper functional. Besides this, $\Psi_{2}$ is convex, sequentially weakly lower semicontinuous and $\Psi_{2}(u) \neq-\infty$ for all $0<u \in W_{0}^{1, \Phi}(\Omega)$.

Proof. First, note that $0 \leq G(x, u) \leq+\infty$ in $\Omega$ for all $u \in W_{0}^{1, \Phi}(\Omega)$, so $\Psi_{2}(u) \neq-\infty$. Moreover, if $u_{0} \in W_{0}^{1, \Phi}(\Omega)$ is a solution of $(S)$, then $u_{0} \in$ $\operatorname{Dom}\left(\Psi_{2}\right)$, which proves $\operatorname{Dom}\left(\Psi_{2}\right) \neq \emptyset$. 
The convexity follows directly from the definition of $\Psi_{2}$. Finally, by the Fatou's lemma, we conclude that $\Psi_{2}$ is sequentially weakly lower semicontinuous.

Lemma 2.3. Suppose $\left(\phi_{0}\right)-\left(\phi_{3}\right),(M),\left(f_{1}\right)$ and $\left(f_{3}\right)$ hold. Then, $I$ is a coercive functional.

Proof. First, by the assumption $(M)$ and Lemma 5.1 in [14], we have

$$
\hat{M}(\mathcal{P}(u)) \geq \frac{m_{0}}{\alpha}\|\nabla u\|_{\Phi}^{\alpha \phi_{-}} \text {for all } u \in W_{0}^{1, \Phi}(\Omega) \text { with }\|\nabla u\|_{\Phi} \geq 1 .
$$

Moreover, by taking $\epsilon>0$ small enough, it follows from $\left(f_{1}\right)$ and $\left(f_{3}\right)$ that $F(x, t) \leq C_{1}+\epsilon|t|^{\alpha \phi_{-}}$for all $x \in \Omega, t \in \mathbb{R}$ and for some $C_{1}>0$. Thus, by the embedding $W_{0}^{1, \Phi}(\Omega) \hookrightarrow L^{\alpha \phi_{-}}(\Omega)$, which follows from the hypothesis $\left(\phi_{3}\right)$, we conclude

$$
\Psi_{1}(u) \geq C_{3}\left(\|\nabla u\|_{\Phi}^{\left.\alpha \phi_{-}-1\right)} \text { for all } u \in W_{0}^{1, \Phi}(\Omega) \text { with }\|\nabla u\|_{\Phi} \geq 1\right.
$$

for some $C_{3}>0$. Since $\delta>1$, we have $\Psi_{2}(u) \geq 0$. Thus, after all these information and (6), we conclude $I(u) \rightarrow \infty$ as $\|\nabla u\|_{\Phi} \rightarrow \infty$, that is, $I$ is coercive. This ends the proof.

Lemma 2.4. Suppose $(S)$ admits a solution in $W_{0}^{1, \Phi}(\Omega)$ and the assumptions $\left(\phi_{0}\right)-\left(\phi_{3}\right),(M),\left(f_{1}\right),\left(f_{3}\right)$ hold. Then I satisfies the $(P S)$ condition.

Proof. Let $\left(u_{n}\right) \subset W_{0}^{1, \Phi}(\Omega)$ and $\left(\epsilon_{n}\right) \subset(0, \infty)$ be sequences such that $I\left(u_{n}\right) \rightarrow$ $c \in \mathbb{R}, \epsilon_{n} \rightarrow 0$ and

$$
\begin{aligned}
& \Psi_{1}^{0}\left(u_{n} ; \varphi-u_{n}\right)+\mu\left(\Psi_{2}(\varphi)-\Psi_{2}\left(u_{n}\right)\right) \\
& \quad \geq-\epsilon_{n}\left\|\nabla\left(\varphi-u_{n}\right)\right\|_{\Phi} \text { for all } \varphi \in W_{0}^{1, \Phi}(\Omega) \text { and } n \in \mathbb{N} .
\end{aligned}
$$

It follows from the coercivity of $I$, obtained in the previous lemma, that $\left(u_{n}\right)$ is bounded in $W_{0}^{1, \Phi}(\Omega)$. Thus, passing to a subsequence if necessary, we may assume that $u_{n} \rightarrow u$. So, by Lemmas 2.1-(vii) and 2.2, we obtain that $I$ is sequentially weakly lower semicontinuous, which yields

$$
I(u) \leq \liminf _{n \rightarrow \infty} I\left(u_{n}\right)=c<\infty,
$$

whence $\Psi_{2}(u)<\infty$. So, by taking $\varphi=u$ in (10), we obtain

$$
-\left(-\Psi_{1}\right)^{0}\left(u_{n} ; u_{n}-u\right) \leq \mu\left(\Psi_{2}(u)-\Psi_{2}\left(u_{n}\right)\right)+\epsilon_{n}\left\|\nabla\left(u_{n}-u\right)\right\|_{\Phi} \text { for } n \in \mathbb{N} \text {. }
$$

Therefore, by using the previous inequality and the lower semicontinuity of $\Psi_{2}$, we get

$$
\liminf _{n \rightarrow \infty}\left(-\Psi_{1}\right)^{0}\left(u_{n} ; u_{n}-u\right) \geq 0
$$

which leads to

$$
\begin{aligned}
0 \leq \liminf _{n \rightarrow \infty}\left(-\Psi_{1}\right)^{0}\left(u_{n} ; u_{n}-u\right) & \leq \liminf _{n \rightarrow \infty}\left[\left(-J_{1}\right)^{0}\left(u_{n} ; u_{n}-u\right)+\lambda J_{2}^{0}\left(u_{n} ; u_{n}-u\right)\right] \\
& =\liminf _{n \rightarrow \infty}\left\langle-J_{1}^{\prime}\left(u_{n}\right) ; u_{n}-u\right\rangle+\lambda \lim _{n \rightarrow \infty} J_{2}^{0}\left(u_{n} ; u_{n}-u\right) \\
& =-\limsup _{n \rightarrow \infty}\left\langle J_{1}^{\prime}\left(u_{n}\right) ; u_{n}-u\right\rangle
\end{aligned}
$$


after applying Lemma 2.1-(iv). Thus, Lemma 2.1-(iii) implies that $u_{n} \rightarrow u$ in $W_{0}^{1, \Phi}(\Omega)$ to a subsequence that ends the proof of lemma.

Proposition 2.1. Assume $\left(\phi_{0}\right)-\left(\phi_{3}\right),(M),\left(f_{1}\right)$ and $\left(f_{3}\right)$ hold. Then, any strict local minimum of the functional $\Psi_{1}=J_{1}-\lambda J_{2}$ in the strong topology of $W_{0}^{1, \Phi}(\Omega)$ is so in the weak topology.

Proof. We just need verify that, under these assumptions, the conditions of Theorem $C$ in $[29]$ are fulfilled. Since $W_{0}^{1, \Phi}(\Omega)$ is a reflexive and separable space, $J_{1}$ and $J_{2}$ are sequentially weakly lower semicontinuous and the functional $\Psi_{1}$ is coercive (see $(9)$ ), we just need to check that $J_{1} \in \mathcal{W}_{W_{0}^{1, \Phi}}$, that is,

"if $u_{n} \rightarrow u$ and $\lim _{n \rightarrow \infty} \inf J_{1}\left(u_{n}\right) \leq J_{1}(u)$, then $u_{n} \rightarrow u$ up to a subsequence"

to conclude the proof of the proposition,

In this direction, let us assume $u_{n} \rightarrow u$ and $\lim _{n \rightarrow \infty} \inf J_{1}\left(u_{n}\right) \leq J_{1}(u)$. Since $J_{1}$ is sequentially weakly lower semicontinuous, we have $\lim _{n \rightarrow \infty} J_{1}\left(u_{n}\right)=J_{1}(u)$ for some subsequence, still denoted by $\left(u_{n}\right)$. Thus, from this fact, continuity and monotonicity of $\hat{M}$ in $\mathbb{R}^{+}$, we obtain $\lim _{n \rightarrow \infty} \mathcal{P}\left(u_{n}\right)=\mathcal{P}(u)$. Therefore, by the hypothesis $\left(\phi_{1}\right)$ we can apply [10, Theorem 2.4.11 and Lemma 2.4.17] to conclude that $u_{n} \rightarrow u$ in $W_{0}^{1, \Phi}(\Omega)$. This ends the proof.

Below, let us connect the existence of solution to problem $(S)$ with existence of two local minima to the functional $I$.

Lemma 2.5. Suppose $(S)$ admits a $W_{0}^{1, \Phi}(\Omega)$-solution, $\left(\phi_{0}\right)-\left(\phi_{3}\right),(M)$ and $\left(f_{1}\right)-\left(f_{3}\right)$ hold. Then, for each $\lambda>\lambda^{*}$ there exists $\mu_{\lambda}>0$ such that for $\mu \in\left(0, \mu_{\lambda}\right]$ the functional I has two local minima.

Proof. Fix $\lambda>\lambda^{*}$, where $\lambda^{*}>0$ was defined at (5). Since $\Psi_{1}$ is lower semicontinuous and coercive (see Lemma 2.1-vii) and (9)), there exists a global minimum $u_{0} \in W_{0}^{1, \Phi}(\Omega)$ of $\Psi_{1}$ in $W_{0}^{1, \Phi}(\Omega)$ and, in particular, $\Psi_{1}\left(u_{0}\right) \leq \Psi_{1}(0)=0$. If $\Psi_{1}\left(u_{0}\right)=0$, we would have

$$
J_{1}(u)-\lambda J_{2}(u)=\Psi_{1}(u) \geq \Psi_{1}\left(u_{0}\right)=0 \text { for all } u \in W_{0}^{1, \Phi}(\Omega),
$$

which would yield $\lambda^{*} \geq \lambda$, but this is impossible.

Let us denote by $C>0$ the best embedding constant of $W_{0}^{1, \Phi}(\Omega) \hookrightarrow$ $L^{\alpha \phi_{+}}(\Omega)$ and take $0<\epsilon<\left(m_{0} C^{\alpha \phi_{+}}\right) / \lambda \alpha$. Thus, it follows from the assumptions $\left(f_{2}\right)$ and $\left(f_{3}\right)$ that $F(x, t) \leq \epsilon t^{\alpha \phi_{+}}$for all $t \in(0, m) \cup(M, \infty)$ for some $m>0$ small enough and $M>0$ large enough.

Besides this, if $\|\nabla u\|_{\Phi}<\epsilon^{\prime}$, then we have

$m^{\alpha \phi_{+}}|[m \leq u \leq M]| \leq\left(\int_{[m \leq u \leq M]} u^{\alpha \phi_{+}} d x\right)^{1 / \alpha \phi^{+}} \leq\|u\|_{\alpha \phi_{+}} \leq C\|\nabla u\|_{\Phi} \leq C \epsilon^{\prime}$,

that is, $|[m \leq u \leq M]| \leq C \epsilon^{\prime} / m^{\alpha \phi_{+}}$. 
So, it follows from the above information and assumption $\left(f_{1}\right)$ that

$$
\begin{aligned}
\int_{\Omega} F(x, u) d x & =\int_{[u<m]} F(x, u) d x+\int_{[u>M]} F(x, u) d x+\int_{[m \leq u \leq M]} F(x, u) d x \\
& \leq \epsilon \int_{\Omega \backslash m \leq u \leq M]} u^{\alpha \phi_{+}} d x+\sup _{m \leq t \leq M} F(x, t) \frac{C \epsilon^{\prime}}{m^{\alpha \phi_{+}}} \leq \epsilon \int_{\Omega} u^{\alpha \phi_{+}} d x
\end{aligned}
$$

for some $\epsilon^{\prime}>0$ small enough, which shows $J_{2}(u) \leq \epsilon\|u\|_{\alpha \phi_{+}}^{\alpha \phi^{+}}$for all $u \in$ $W_{0}^{1, \Phi}(\Omega)$ with $\|\nabla u\|_{\Phi} \leq \epsilon^{\prime}$.

Therefore, we obtain from this fact, hypothesis $(M)$ and Lemma 5.1 in (8) that

$$
\Psi_{1}(u) \geq \frac{m_{0}}{\alpha}\|\nabla u\|_{\Phi}^{\alpha \phi_{+}}-\lambda \epsilon\|u\|_{\alpha \phi_{+}}^{\alpha \phi_{+}} \geq \frac{m_{0} C^{\alpha \phi_{+}}}{\alpha}\|u\|_{\alpha \phi_{+}}^{\alpha \phi_{+}}-\lambda \epsilon\|u\|_{\alpha \phi_{+}}^{\alpha \phi_{+}}>0=\Psi_{1}(0)
$$

holds, whenever $\|\nabla u\|_{\Phi}<\epsilon^{\prime}$ with $\epsilon^{\prime}>0$ such above, that is, 0 is a strict local minimum of $\Psi_{1}$ in the strong topology. Hence, we obtain from Proposition 2.1 that 0 is a local strict minimum of $\Psi_{1}$ in the weak topology as well, i.e, there exists a weak neighborhood $V_{w}$ of 0 such that

$$
0=\Psi_{1}(0)<\Psi_{1}(u) \text { for all } u \in V_{w} \backslash\{0\} .
$$

After these information and the assumption that the problem $(S)$ admits a solution in $W_{0}^{1, \Phi}(\Omega)$, we are able to follow the same strategy of the proof of Theorem 1.1 in [12] to build disjoint open sets $D_{1}$ and $D_{2}$, in the strong topology, such that $0 \in D_{1}, u_{0} \in D_{2}$ and to find $\tilde{\omega}_{i} \in D_{i}$ such that $\tilde{\omega}_{1}$ and $\tilde{\omega}_{2}$ are distinct local minima of $I$. This ends the proof.

By applying Corollary 2.1 of [23] for functional of the type locally Lipschiz plus convex (it is a version of Corollary 3.3 in [32] that considers functional of the type $C^{1}$ plus convex), Lemmas 2.4 and 2.5, we have the following property.

Corollary 2.1. Suppose $\left(\phi_{0}\right)-\left(\phi_{3}\right),(b),(M)$ and $\left(f_{1}\right)-\left(f_{3}\right)$ hold. In addition, assume that Problem $(S)$ admits a $W_{0}^{1, \Phi}(\Omega)$-solution. Then, for each $\lambda>\lambda^{*}$ there exists $\mu_{\lambda}>0$ such that for $\mu \in\left(0, \mu_{\lambda}\right]$ the functional $I$ has three critical points, being two of them local minima and the other one a mountain pass point to the functional $I$.

\section{Proof of Theorem 1.1}

Before starting the proof of Theorem 1.1, let us prove the two below lemmas.

Lemma 3.1. (Multivalued solutions) Assume $\left(\phi_{0}\right)-\left(\phi_{3}\right),(M),\left(f_{0}\right),\left(f_{1}\right), 0<$ $b \in L^{1}(\Omega)$ and $u \in W_{0}^{1, \Phi}(\Omega)$ be a critical point of $I$. Then:

(i) $u>0$ a.e. in $\Omega$ and there exist $a \eta \in \partial \Psi_{2}(u)$ and $a \rho \in[f(x, u(x)-0$, $f(x, u(x)+0)] \subset L^{\widetilde{H}}(\Omega)$ such that

$(M \circ \mathcal{P})(u) \int_{\Omega} a(|\nabla u|) \nabla u \nabla \varphi d x=\mu\langle\eta, \varphi\rangle+\lambda \int_{\Omega} \rho \varphi d x$ for all $\varphi \in W_{0}^{1, \Phi}(\Omega)$, 
where $\partial \Psi_{2}(u)$ stands for the subdifferential of the convex functional $\Psi_{2}$ at $u$,

(ii) $b u^{-\delta} \varphi \in L^{1}(\Omega)$ for any $\varphi \in W_{0}^{1, \Phi}(\Omega)$. Besides this,

$$
\partial \Psi_{2}(u)=\{\eta\} \text { and }\langle\eta, \varphi\rangle=-\int_{\Omega} b u^{-\delta} \varphi d x \text { for all } \varphi \in W_{0}^{1, \Phi}(\Omega) .
$$

In particular, the Eq. (11) turns into

$(M \circ \mathcal{P})(u) \int_{\Omega} a(|\nabla u|) \nabla u \nabla \varphi d x=\int_{\Omega}\left[\mu b u^{-\delta}+\rho\right] \varphi d x$ for all $\varphi \in W_{0}^{1, \Phi}(\Omega)$,

(iii) there exists a $C>0$, dependent on $u$, such that $u(x) \geq C d(x)$ for $x \in \Omega$, (iv) $\rho+b u^{-\delta} \in L_{\mathrm{loc}}^{2}(\Omega)$ if in addition $b \in L_{\mathrm{loc}}^{2}(\Omega)$.

Proof of $(i)$. Since $u$ is a critical point of $I$ (see (7)), in particular, we have $u \in \operatorname{Dom}\left(\Psi_{2}\right)$, which implies $\int_{\Omega}|G(x, u)| d x<\infty$, that is, $G(\cdot, u(\cdot))$ is finite a.e. in $\Omega$. Therefore, by the definition of $G$, we have $u>0$ a.e in $\Omega$.

Again, by $u \in W_{0}^{1, \Phi}(\Omega)$ be a critical point of $I$, it follows from [4, Proposition 2.183], that

$$
0 \in-(M \circ \mathcal{P})(u) \Delta_{\Phi} u-\lambda \partial J_{2}(u)+\mu \partial \Psi_{2}(u),
$$

where $\partial J_{2}(u)$ stands for the generalized gradient of the locally Lipschiz continuous functional $J_{2}$ at $u$. Thus, there exist $\rho \in \partial J_{2}(u)$ and $\eta \in \partial \Psi_{2}(u)$ such that

$$
\left\langle-(M \circ \mathcal{P})(u) \Delta_{\Phi} u, v\right\rangle=\lambda\langle\rho, v\rangle-\mu\langle\eta, v\rangle \text { for all } v \in W_{0}^{1, \Phi}(\Omega) .
$$

So, it follows from Lemma 2.1-(ii) that there exists a unique $\rho \in L^{\tilde{H}}(\Omega)$, with $\rho \in[f(x, u(x)-0), f(x, u(x)+0)]$, such that the equality (11) holds true. This ends the proof of $(i)$.

Let us prove $(i i)$. By (11) and $\rho \geq 0$, we have

$$
(M \circ \mathcal{P})(u) \int_{\Omega} a(|\nabla u|) \nabla u \nabla \varphi d x \geq-\mu\langle\eta, \varphi\rangle \text { for all } 0 \leq \varphi \in W_{0}^{1, \Phi}(\Omega),
$$

which implies, by definition of $\eta \in \partial \Psi_{2}(u)$, that

$$
\begin{aligned}
& (M \circ \mathcal{P})(u) \int_{\Omega} a(|\nabla u|) \nabla u \nabla \varphi d x \\
& \quad \geq \mu \int_{\Omega}\left[\frac{G(x, u)-G(x, u+t \varphi)}{t}\right] d x \text { for all } 0 \leq \varphi \in W_{0}^{1, \Phi}(\Omega) .
\end{aligned}
$$

Hence, by $u>0$ a.e. in $\Omega$ and Fatou's lemma, we obtain

$$
\begin{aligned}
\mu \int_{\Omega} b(x) u^{-\delta} \varphi d x & \leq \liminf _{t \rightarrow 0^{+}} \frac{1}{-\delta+1} \int_{\Omega} \mu b(x)\left(\frac{(u+t \varphi)^{-\delta+1}-u^{-\delta+1}}{t}\right) d x \\
& \leq M(\mathcal{P}(u)) \int_{\Omega} a(|\nabla u|) \nabla u \nabla \varphi d x<\infty \text { for all } 0 \leq \varphi \in W_{0}^{1, \Phi}(\Omega),
\end{aligned}
$$


that proves that $b u^{-\delta} \varphi \in L^{1}(\Omega)$ for any $\varphi \in W_{0}^{1, \Phi}(\Omega)$. To finish the proof of (ii), let $\eta \in \partial \Psi_{2}(u)$. Then for $\epsilon \in(0,1)$, we have

$$
\Psi_{2}(u-\epsilon u)-\Psi_{2}(u) \geq-\epsilon\langle\eta, u\rangle,
$$

which can be rewritten as

$$
\frac{(1-\epsilon)^{-\delta+1}-1}{(\delta-1) \epsilon} \int_{\Omega} b(x) u^{-\delta+1} d x \geq-\langle\eta, u\rangle .
$$

So, by doing $\epsilon \rightarrow 0^{+}$in the previous inequality, we obtain

$$
\int_{\Omega} b(x) u^{-\delta+1} d x \geq-\langle\eta, u\rangle .
$$

On the other hand, again by the fact that $\eta \in \partial \Psi_{2}(u)$, one has

$$
\begin{aligned}
\langle\eta, \varphi\rangle & \leq \frac{\Psi_{2}(u+\epsilon \varphi)-\Psi_{2}(u)}{\epsilon} \\
& =\frac{1}{-\delta+1} \int_{\Omega} b(x)\left(\frac{u^{-\delta+1}-(u+\epsilon \varphi)^{-\delta+1}}{\epsilon}\right) d x
\end{aligned}
$$

for all $0 \leq \varphi \in W_{0}^{1, \Phi}(\Omega)$ and $\epsilon>0$ given, which yields

$$
-\int_{\Omega} b(x) u^{-\delta} \varphi d x \geq\langle\eta, \varphi\rangle
$$

after using Fatou's lemma.

By taking $\varphi=u$ in (16) and combining this with (15), we obtain

$$
\langle\eta, u\rangle=-\int_{\Omega} b(x) u^{-\delta+1} d x .
$$

Besides this, by letting $\varphi \in W_{0}^{1, \Phi}(\Omega)$, testing (16) with $(u+\epsilon \varphi)^{+}$and using (17), we get

$$
-\epsilon \int_{\Omega} b(x) u^{-\delta} \varphi d x \geq \epsilon\langle\eta, \varphi\rangle-\left\langle\eta, u \cdot \chi_{[u+\epsilon \varphi \leq 0]}\right\rangle-\epsilon\left\langle\eta, \varphi \cdot \chi_{[u+\epsilon \varphi \leq 0]}\right\rangle,
$$

which lead us to

$$
-\int_{\Omega} b(x) u^{-\delta} \varphi d x \geq\langle\eta, \varphi\rangle-\left\langle\eta, \varphi \cdot \chi_{[u+\epsilon \varphi \leq 0]}\right\rangle,
$$

due (16), that is, $-\left\langle\eta, u \cdot \chi_{[u+\epsilon \varphi \leq 0]}\right\rangle \geq 0$.

By using that $|[u+\epsilon \varphi \leq 0]| \rightarrow 0$ as $\epsilon \rightarrow 0$, the inequality (18) yields

$$
-\int_{\Omega} b(x) u^{-\delta} \varphi d x \geq\langle\eta, \varphi\rangle, \text { for all } \varphi \in W_{0}^{1, \Phi}(\Omega),
$$

that is,

$$
\langle\eta, \varphi\rangle=-\int_{\Omega} b(x) u^{-\delta} \varphi d x, \text { for all } \varphi \in W_{0}^{1, \Phi}(\Omega) .
$$

This ends the proof of item $(i i)$.

Now, we are ready to prove $(i i i)$. First, let us denote by $c_{0}:=M(\mathcal{P}(u))>0$ and consider the problem

$$
-\Delta_{\Phi} v=\frac{\mu}{c_{0}} b_{1}(x)(v+1)^{-\delta} \text { in } \Omega, v=0 \text { on } \partial \Omega,
$$


where $b_{1}(x)=\min \{1, b(x)\}$. We know from Lemmas 4.2 and 5.1 in [15] that there exist a unique solution of (19), say $\tilde{u}_{1} \in W_{0}^{1, \Phi}(\Omega)$, and $C=C_{u}>0$ such that $\tilde{u}_{1} \geq C d$ in $\Omega$.

On the other hand, we obtain from (14) that

$$
\begin{aligned}
\int_{\Omega} a(|\nabla u|) \nabla u \nabla \varphi d x & \geq \int_{\Omega} \frac{\mu}{c_{0}} b(x) u^{-\delta} \varphi d x \\
& \geq \int_{\Omega} \frac{\mu}{c_{0}} b_{1}(x)(u+1)^{-\delta} \varphi d x \text { for all } 0 \leq \varphi \in W_{0}^{1, \Phi}(\Omega),
\end{aligned}
$$

that is, $u$ is a supersolution for the problem (19). Hence,

$$
\begin{aligned}
0 & \leq \int_{\Omega}\left(a\left(\left|\nabla \tilde{u}_{1}\right|\right) \nabla \tilde{u}_{1}-a(|\nabla u|) \nabla u\right) \nabla\left(\tilde{u}_{1}-u\right)^{+} d x \\
& \leq \frac{\mu}{c_{0}} \int_{\Omega} b_{1}(x)\left(\left(\tilde{u}_{1}+1\right)^{-\delta}-(u+1)^{-\delta}\right)\left(\tilde{u}_{1}-u\right)^{+} d x \leq 0,
\end{aligned}
$$

which implies that $C d \leq \tilde{u}_{1} \leq u$ in $\Omega$ and this proves $(i i i)$.

Let us prove $(i v)$. By $\left(f_{1}\right)$ and property $\tilde{H}^{-1}(H(t)) \leq 2 \tilde{h}^{-1}(t)=2 h(t)$ for all $t \in \mathbb{R}$ (the equality is due $h$ being continuous), we obtain

$$
|\rho| \leq a_{2} \widetilde{H}^{-1} \circ H\left(a_{3}(|u|+|\varphi|)\right)+a_{1} \leq 2 a_{2} h\left(a_{3}|u|\right)+a_{1} \leq C\left(1+u^{h_{+}-1}\right),
$$

for some $C>0$, where the last inequality is a consequence of $(3)$ in $\left(f_{1}\right)$. Hence, we obtain from $(20), W_{0}^{1, \Phi}(\Omega) \hookrightarrow L^{\phi_{-}^{*}}(\Omega)$ and $h_{+} \leq \phi_{-}^{*} / 2+1$ that $\rho \in L_{\text {loc }}^{2}(\Omega)$. So, combining the fact that $\rho \in L_{\text {loc }}^{2}(\Omega)$ together with $(i)$ above, the proof of $(i v)$ follows. This ends the proof of lemma.

Lemma 3.2. (Almost everywhere solutions) Assume $\left(\phi_{0}\right)-\left(\phi_{3}\right),(M),\left(f_{0}\right)$, $\left(f_{1}\right)$ and $b d^{-\delta} \in L^{\tilde{H}}(\Omega)$. Let $u \in W_{0}^{1, \Phi}(\Omega)$ be a critical point of $I$ and $\rho \in$ $L^{\widetilde{H}}(\Omega)$ as in Lemma 3.1. Then:

$(i)-(M \circ \mathcal{P})(u) \Delta_{\Phi} u \in\left(L^{H}(\Omega)\right)^{\prime}$,

(ii) there exists a unique representative of $-(M \circ \mathcal{P})(u) \Delta_{\Phi} u$ in $L^{\widetilde{H}}(\Omega)$, still denoted by $-(M \circ \mathcal{P})(u) \Delta_{\Phi} u$, such that

$$
-(M \circ \mathcal{P})(u) \Delta_{\Phi} u=\lambda \rho+\mu b u^{-\delta} \text { a.e. in } \Omega .
$$

Proof of $(i)$ We have from (13) that

$$
\left\langle-(M \circ \mathcal{P})(u) \Delta_{\Phi} u, \varphi\right\rangle=\lambda\langle\rho, \varphi\rangle-\mu\langle\eta, \varphi\rangle \text { for all } \varphi \in W_{0}^{1, \Phi}(\Omega),
$$

where $\eta \in \partial \Psi_{2}(u) \subset\left(W_{0}^{1, \Phi}(\Omega)\right)^{\prime}$ and $\rho \in \partial J_{2}(u) \subset\left(L^{H}(\Omega)\right)^{\prime}$ with this last inclusion due to the Lemma 2.1-(ii). Since $b d^{-\delta} \in L^{\tilde{H}}(\Omega)$, we obtain from Lemma 3.1- $(i i)$ and $($ iii $)$ that $\eta \in\left(L^{H}(\Omega)\right)^{\prime}$ as well. Thus, we obtain from these information and $\overline{W_{0}^{1, \Phi}(\Omega)}\left\|_{H}=\right\|^{H}(\Omega)$ that $-(M \circ \mathcal{P})(u) \Delta_{\Phi} u \in\left(L^{H}(\Omega)\right)^{\prime}$. This proves $(i)$. 
Let us prove $(i i)$. It follows from item $(i)$ and the Riesz theorem for Orlicz spaces that there exist a unique element in $L^{\widetilde{H}}(\Omega)$, still denoted by $-(M \circ \mathcal{P})(u) \Delta_{\Phi} u$, such that

$$
\left\langle-M \circ \mathcal{P}(u) \Delta_{\Phi} u, \varphi\right\rangle=\int_{\Omega}\left(-M \circ \mathcal{P}(u) \Delta_{\Phi} u\right) \varphi d x \text { for all } \varphi \in W_{0}^{1, \Phi}(\Omega),
$$

which implies by (12) that

$$
\int_{\Omega}\left(-M \circ \mathcal{P}(u) \Delta_{\Phi} u-\lambda \rho-\mu b u^{-\delta}\right) \varphi=0 \text { for all } \varphi \in W_{0}^{1, \Phi}(\Omega) .
$$

This ends the proof of lemma.

Proof of Theorem 1.1-Conclusion. The proof of item $(i)$ is inspired on ideas from [9], while for the proof of $(i i)$ we borrow strategies from [22]. The item $($ iii $)-(v)$ are consequences of Lemmas 3.1 and 3.2.

Proof of $(i)$ : We just consider the case when $u$ is a local minimum for $I$. Similar arguments work when $u$ is a local maximum for $I$. In this case, it is readily that

$$
\begin{aligned}
\lambda \int_{\Omega} \frac{F(x, u+\epsilon \varphi)-F(x, u)}{\epsilon} d x & -\mu \int_{\Omega} \frac{G(x, u+\epsilon \varphi)-G(x, u)}{\epsilon} \varphi d x \\
& \leq \int_{\Omega} \frac{\hat{M}(\mathcal{P}(u+\epsilon \varphi))-\hat{M}(\mathcal{P}(u))}{\epsilon} d x
\end{aligned}
$$

holds for any $\varphi \in W_{0}^{1, \Phi}(\Omega)$ and any $\epsilon>0$ given.

Below, let us consider two cases. First, fix $0 \leq \varphi \in \mathbf{C}_{0}^{\infty}(\Omega)$. So, we obtain from Lebourg's theorem that there exist $t_{0}(x) \in(0,1)$ and $\xi_{\epsilon} \in \partial F\left(x, u+t_{0} \epsilon \varphi\right)$ such that

$$
\frac{F(x, u+\epsilon \varphi)-F(x, u)}{\epsilon}=\xi_{\epsilon} \varphi
$$

for each $x \in \Omega$.

By using $\left(f_{1}\right)$, we are able to estimate $\xi_{\epsilon}$ by

$$
\left|\xi_{\epsilon}\right| \leq a_{2} \widetilde{H}^{-1} \circ H\left(a_{3}(|u|+|\varphi|)\right)+a_{1}:=g,
$$

where $g \in L^{1}(\Omega)$ is independent of $\epsilon>0$. Hence, coming back to (23), we obtain

$$
\left|\frac{F(x, u+\epsilon \varphi)-F(x, u)}{\epsilon}\right| \leq g \varphi \in L^{1}(\Omega)
$$

for every $\epsilon>0$ small enough.

Besides this, the right derivative of $F(x, \cdot)$ at $u$ is given by

$$
\lim _{\epsilon \rightarrow 0^{+}} \frac{F(x, u+\epsilon \varphi)-F(x, u)}{\epsilon}=f(x, u(x)+0) \varphi \text { a.e. } x \in \Omega,
$$

because $\varphi \geq 0$. 
So, we are in position to apply the Lebesgue theorem, combined with Fatou's lemma and Lemma 3.2, in (22) to show that

$$
\begin{aligned}
& \int_{\Omega}\left(\lambda f(x, u(x)+0)+\mu \frac{b(x)}{u^{\delta}}\right) \varphi d x \leq(M \circ \mathcal{P})(u) \int_{\Omega} \phi(|\nabla u|) \nabla u \nabla \varphi d x \\
& \quad=\int_{\Omega}-(M \circ \mathcal{P})(u) \Delta_{\Phi} u \varphi d x
\end{aligned}
$$

holds for any $0 \leq \varphi \in W_{0}^{1, \Phi}(\Omega)$, that is,

$$
-(M \circ \mathcal{P})(u) \Delta_{\Phi} u(x) \geq \lambda f(x, u(x)+0)+\mu \frac{b(x)}{u^{\delta}} \text { a.e. } x \in \Omega .
$$

On the other hand, it follows from (21) and Lemma 2.1-(ii) that

$$
-(M \circ \mathcal{P})(u) \Delta_{\Phi} u(x) \leq \lambda f(x, u(x)+0)+\mu \frac{b(x)}{u^{\delta}} \text { a.e. } x \in \Omega,
$$

due to the fact that $u$ is a critical point of $I$.

After these two inequalities, we obtain

$$
-(M \circ \mathcal{P})(u) \Delta_{\Phi} u=\lambda f(x, u(x)+0)+\mu \frac{b(x)}{u^{\delta}}, \text { a.e. } x \in \Omega .
$$

Secondly, let us fix $\varphi \in \mathbf{C}_{0}^{\infty}(\Omega)$ with $\varphi \leq 0$. By similar arguments as those done to prove the case $\varphi \geq 0$, we are able to show that

$$
-(M \circ \mathcal{P})(u) \Delta_{\Phi} u=\lambda f(x, u(x)-0)+\mu \frac{b(x)}{u^{\delta}} \text { a.e. } x \in \Omega .
$$

holds.

Finally, if meas $\{x \in \Omega: u(x)=\tilde{a}\}>0$, then it would have from (24) and (25) that

$$
f(x, a-0)=f(x, \tilde{a}+0) \text { a.e. } x \in\{x \in \Omega: u(x)=\tilde{a}\},
$$

but this is impossible by $\left(f_{0}\right)$ so meas $\{x \in \Omega: u(x)=\tilde{a}\}=0$. This ends the proof of $(i)$.

Proof of $(i i)$ : Since $u \in W_{0}^{1, \Phi}(\Omega)$ is a critical point of $I$, we obtain from Lemmas 3.2-(ii) that

$$
-(M \circ \mathcal{P})(u) \Delta_{\Phi} u=\lambda \rho+\mu b u^{-\delta}:=h(x) \text { a.e. in } \Omega .
$$

with $\rho \in[f(x, u-0), f(x, u+0)]$. So, it follows from Lemma 3.1 and $[6$, Theorem 2.1], that

$$
a(|\nabla u|)|\nabla u| \in W_{l o c}^{1,2}(\Omega)
$$

Besides this, we have

$$
\left|\nabla\left(\frac{a(|\nabla u|)|\nabla u|}{\epsilon+a(|\nabla u|)|\nabla u|}\right)\right|=\frac{\epsilon|\nabla(a(|\nabla u|)|\nabla u|)|}{[\epsilon+a(|\nabla u|)|\nabla u|]^{2}} \leq \frac{1}{\epsilon}|\nabla(a(|\nabla u|)|\nabla u|)|,
$$

which shows that

$$
\frac{a(|\nabla u|)|\nabla u|}{\epsilon+a(|\nabla u|)|\nabla u|} \in W_{l o c}^{1,2}(\Omega)
$$


for each $\epsilon>0$ given and so

$$
\frac{a(|\nabla u|)|\nabla u|}{\epsilon+a(|\nabla u|)|\nabla u|} \varphi \in W_{0}^{1,2}(\Omega)
$$

can be used as a test function for any $\epsilon>0$ and any $\varphi \in \mathbf{C}_{0}^{\infty}(\Omega)$ given.

By doing this, we get from (26) that

$$
\begin{aligned}
\int_{\Omega} & h(x)\left(\frac{a(|\nabla u|)|\nabla u|}{\epsilon+a(|\nabla u|)|\nabla u|} \varphi\right) \\
= & (M \circ \mathcal{P})(u) \int_{\Omega} a(|\nabla u|) \nabla u \nabla\left(\frac{a(|\nabla u|)|\nabla u|}{\epsilon+a(|\nabla u|)|\nabla u|} \varphi\right) d x \\
= & (M \circ \mathcal{P})(u) \int_{\Omega} a(|\nabla u|) \frac{a(|\nabla u|)|\nabla u|}{\epsilon+a(|\nabla u|)|\nabla u|} \nabla u \nabla \varphi d x \\
& \quad+(M \circ \mathcal{P})(u) \int_{\Omega} a(|\nabla u|) \varphi_{\frac{\epsilon}{[\epsilon+a(|\nabla u|)|\nabla u|]^{2}}} \nabla(a(|\nabla u|)|\nabla u|) \nabla u d x .
\end{aligned}
$$

Since,

$$
\begin{aligned}
& \left|a(|\nabla u|) \varphi \frac{\epsilon}{[\epsilon+a(|\nabla u|)|\nabla u|]^{2}} \nabla(a(|\nabla u|)|\nabla u|) \nabla u\right| \\
& \quad \leq|\varphi| \frac{\epsilon^{2}+(a(|\nabla u|)|\nabla u|)^{2}}{2[\epsilon+a(|\nabla u|)|\nabla u|]^{2}}|\nabla(a(|\nabla u|)|\nabla u|)| \\
& \quad \leq \frac{1}{2}|\varphi \nabla(a(|\nabla u|)|\nabla u|)|
\end{aligned}
$$

holds for any $\epsilon>0$, we are able to apply Lebesgue's theorem to the equalities in (27) to infer that

$$
\begin{aligned}
\int_{\Omega \backslash\{\nabla u \neq 0\}} h \varphi d x & =(M \circ \mathcal{P})(u) \int_{\Omega \backslash\{\nabla u \neq 0\}} a(|\nabla u|) \nabla u \nabla \varphi \\
& =(M \circ \mathcal{P})(u) \int_{\Omega} a(|\nabla u|) \nabla u \nabla \varphi=\int_{\Omega} h \varphi d x
\end{aligned}
$$

holds, which lead us to have $h(x)=0$ a.e. in $\{x \in \Omega: \nabla u=0\}$. As we already know from Lemma 3.1-(ii) that $h(x)>0$ in $\Omega$, we obtain that $\operatorname{meas}\{x \in \Omega: \nabla u=0\}=0$. So, it follows from Morey-Stampacchia's theorem that $\{x \in \Omega: u=c\} \subset\{x \in \Omega: \nabla u=0\}$ for any real constant $c$ given, which shows that meas $\{x \in \Omega: u=c\}=0$.

So, as a consequence of $(i)$ and $(i i)$ above, $\rho(x)=f(x, u(x))$ if $u(x) \neq a$ and $\rho(x) \in[f(x, a-0), f(x, a+0)]$ if $u(x)=a$, we obtain that $\rho(x)=f(x, u(x))$ a.e. in $\Omega$. Finally, by applying Lemma 3.1, we have (iii) and (iv), while Lemma 3.2 implies $(v)$. This ends the proof.

As a consequence of our above approach, we proved an abstract result of regularity that is itself important.

Theorem 3.1. Assume $(M)$ and $\left(\phi_{0}\right)-\left(\phi_{2}\right)$ hold. Let $h \in L_{l o c}^{2}(\Omega)$ be such that $h \neq 0$ a.e. in $\Omega$. If $u \in W_{0}^{1, \Phi}(\Omega)$ is a weak solution of

$$
\text { (Q) } \begin{cases}-M\left(\int \Phi(|\nabla u|) d x\right) \Delta_{\Phi} u=h(x) & \text { in } \Omega, \\ u>0 & \text { in } \Omega, u=0 \text { on } \partial \Omega,\end{cases}
$$

then meas $\{x \in \Omega:|\nabla u(x)|=0\}=0$. In particular, meas $\{x \in \Omega: u(x)=$ $c\}=0$ for each $c>0$. 


\section{Proof of Theorem 1.2}

In this section, let us begin proving the equivalences among $(i),(i i)$ and $(i i i)$. To prove $(i \Longrightarrow i i)$, we borrow ideas from Yijing [31], who treated this situation in the context of homogeneous operators. The principal difficulty in doing this is to find appropriated assumptions under the $\mathrm{N}$-function $\Phi$ to become possible to obtain compactness results for minimizing sequences on Nehari sets type, while the main obstacles to prove $(i i \Longrightarrow i i i)$ were already got over in the last section. The $(i i i \Longrightarrow i)$ is immediately. We will end this section ensuring that the discontinuity of the nonlinearity $f(x, \cdot)$ may be attained.

Let us begin by defining the set

$$
\mathcal{A}:=\left\{u \in W_{0}^{1, \Phi}(\Omega): \int_{\Omega} b(x)|u|^{1-\delta} d x<\infty\right\},
$$

which turns well-defined the functional

$$
J(u)=\int_{\Omega} \Phi(|\nabla u|) d x+\frac{1}{\delta-1} \int_{\Omega} b(x)|u|^{1-\delta} d x, u \in \mathcal{A}
$$

and the subsets

$$
\mathcal{N}:=\left\{u \in W_{0}^{1, \Phi}(\Omega): \int_{\Omega}\left(a(|\nabla u|)|\nabla u|^{2}-b(x)|u|^{1-\delta}\right) d x \geq 0\right\}
$$

and

$$
\mathcal{N}^{*}:=\left\{u \in W_{0}^{1, \Phi}(\Omega): \int_{\Omega}\left(a(|\nabla u|)|\nabla u|^{2}-b(x)|u|^{1-\delta}\right) d x=0\right\} .
$$

Lemma 4.1. Assume $\left(\phi_{1}\right)$ and $\mathcal{A} \neq \emptyset$. Then $\mathcal{N}^{*}$ and $\mathcal{N}$ are non-empty sets and $\mathcal{N}$ is unbounded set.

Proof. Take $u \in \mathcal{A}$. So, it follows from $\left(\phi_{1}\right)$ and Lemma 5.1 in [14], that

$$
\begin{aligned}
\int_{\Omega} \phi(t|\nabla u|)|\nabla u| d x & \geq \frac{\phi_{-}}{t} \int_{\Omega} \Phi(t|\nabla u|) d x \\
& \geq \min \left\{t^{\phi_{-}-1}, t^{\phi_{+}-1}\right\} \phi_{-} \int_{\Omega} \Phi(|\nabla u|) d x
\end{aligned}
$$

and

$$
\begin{aligned}
\int_{\Omega} \phi(t|\nabla u|)|\nabla u| d x & \leq \frac{\phi_{+}}{t} \int_{\Omega} \Phi(t|\nabla u|) d x \\
& \leq \max \left\{t^{\phi_{-}-1}, t^{\phi_{+}-1}\right\} \phi_{-} \int_{\Omega} \Phi(|\nabla u|) d x
\end{aligned}
$$

hold for $t>0$ large enough.

So, we obtain from (28) and (29) that $\sigma^{\prime}(t) \rightarrow \infty$ as $t \rightarrow \infty$ and $\sigma^{\prime}(t) \rightarrow$ $-\infty$ as $t \rightarrow 0^{+}$. Besides this, we have from $\left(\phi_{1}\right)$ again that $\sigma^{\prime \prime}(t)>0$ for all $t>0$, where

$$
\sigma(t):=J(t u)=\int_{\Omega} \Phi(t|\nabla u|) d x+\frac{t^{1-\delta}}{\delta-1} \int_{\Omega} b(x)|u|^{1-\delta} d x, t>0
$$


and so there exists a unique $t_{*}=t_{*}(u)$ (which is a global minimum of $\sigma$ ) such that $\sigma^{\prime}\left(t_{*}\right)=0$. This shows that $t_{*} u \in \mathcal{N}^{*}$. As another consequence of the above information, we have that $\sigma^{\prime}(t) \geq 0$ for all $t>0$ large enough, that is, $t u \in \mathcal{N}$ for all $t>0$ large enough. In particular, $\mathcal{N}$ is unbounded as well. This ends the proof.

By using similar ideas as done Yijing [31] for the homogeneous case, we are able to prove the below lemma in the context of non-local and nonhomogeneous operator.

Lemma 4.2. Assume $\left(\phi_{1}\right)$ and $\mathcal{A} \neq \emptyset$. Then:

(i) the set $\mathcal{N}$ is strong closed,

(ii) 0 is not an accumulation point of $\mathcal{N}$.

To complete our basics tools to prove Theorem 1.2, let us prove the below lemma that is interesting itself.

Lemma 4.3. Assume that $0<b \in L^{1}(\Omega),\left(\phi_{0}\right)$ and $(M)$ hold. Let $g: \Omega \times$ $(0, \infty) \longrightarrow \mathbb{R}$ be a Carathéodory function such that

$$
(g(x, s)-g(x, t))(s-t) \leq 0 \text { for all } s, t>0 .
$$

Then the problem

$$
\left\{\begin{array}{l}
-M\left(\int_{\Omega} \Phi(|\nabla u|) d x\right) \Delta_{\Phi} u=\lambda \frac{b(x)}{u^{\delta}}+g(x, u), \text { in } \Omega \\
u>0 \text { in } \Omega, u=0 \text { on } \partial \Omega
\end{array}\right.
$$

has at most one solution in $W_{0}^{1, \Phi}(\Omega)$.

Proof. First, we note that the fact of $M$ being non-increasing implies that $\hat{M}$ is convex. With similar arguments together with the hypotheses $\left(\phi_{0}\right)$, we show that $\Phi$ convex as well. These facts and the hypotheses $(M)$ lead us to infer that the functional

$$
J_{1}(u):=\hat{M}\left(\int_{\Omega} \Phi(|\nabla u|) d x\right), u \in W_{0}^{1, \Phi}(\Omega)
$$

is convex as well.

Let $u, v \in W_{0}^{1, \Phi}(\Omega)$ be two different solutions of the problem (31). So, it follows from (30) and the convexity of $J_{1}$, that

$$
\begin{aligned}
0 \leq\left\langle J_{1}^{\prime}(u)-J_{1}^{\prime}(v), u-v\right\rangle & =\lambda \int_{\Omega}\left(\frac{b}{u^{\delta}}-\frac{b}{v^{\delta}}\right)(u-v) d x+\int_{\Omega}(g(x, u)-g(x, v))(u-v) d x \\
& \leq \lambda \int_{\Omega}\left(\frac{b}{u^{\delta}}-\frac{b}{v^{\delta}}\right)(u-v) d x<0,
\end{aligned}
$$

where the last inequality follows from $b, \delta>0$. This is impossible and so the proof of Lemma 4.3 is done.

Proof of Theorem 1.2-Conclusion. We begin proving the first implication.

Proof of $(i) \Longrightarrow(i i)$. First, we note that the assumption $(i)$ implies that $\mathcal{A} \neq \emptyset$. So, it follows from Lemmas 4.1 and 4.2 that $\mathcal{N}$ is a nonempty complete metric space. Moreover, by Lemmas 2.1 (vi), Lemma 2.2 and the fact that

$$
J(u) \geq \min \left\{\|\nabla u\|_{\Phi}^{\phi_{-}},\|\nabla u\|_{\Phi}^{\phi_{+}}\right\}
$$


we have that $J$ is lower semicontinuous and bounded below. Thus, by the Ekeland Variational Principle there exists a minimizing sequence $\left(u_{n}\right) \subset \mathcal{N}$ to $J$ constrained to $\mathcal{N}$ such that:

(i) $J\left(u_{n}\right) \leq \inf _{\mathcal{N}} J+\frac{1}{n}$

(ii) $J\left(u_{n}\right) \leq J(w)+\frac{1}{n}\left\|\nabla\left(u_{n}-w\right)\right\|_{\Phi}, \forall w \in \mathcal{N}$.

Besides this, we may assume $u_{n}(x)>0$ a.e in $\Omega$, because $J\left(\left|u_{n}\right|\right)=J\left(u_{n}\right)$ and if we assume that $u_{n}=0$ in a measurable set $\Omega_{0} \subset \Omega$, with $\left|\Omega_{0}\right|>0$, then we would have from $u_{n} \in \mathcal{N}, b(x)>0$ a.e in $\Omega$ and reverse Hölder inequality that

$\infty>\phi_{+} \int_{\Omega} \Phi\left(\left|\nabla u_{n}\right|\right) d x \geq \int_{\Omega_{0}} b(x) u_{n}^{1-\delta} \geq\left(\int_{\Omega_{0}} b(x)^{1 / \delta} d x\right)^{\delta}\left(\int_{\Omega_{0}}\left|u_{n}\right| d x\right)^{1-\delta}=\infty$

which is an absurd. Thus, $u_{n}(x)>0$ a.e in $\Omega$.

Since $J\left(u_{n}\right) \rightarrow \inf _{\mathcal{N}} J \geq 0$, we have

$$
\min \left\{\left\|\nabla u_{n}\right\|_{\Phi}^{\phi_{-}},\left\|\nabla u_{n}\right\|_{\Phi}^{\phi_{+}}\right\} \leq \int_{\Omega} \Phi\left(\left|\nabla u_{n}\right|\right) d x \leq \epsilon+\inf _{\mathcal{N}} J
$$

for all $n$ large enough, which implies that $\left(u_{n}\right)$ is bounded. As a consequence of this, we have that

$$
\left\{\begin{array}{l}
u_{n} \rightarrow u_{*} \text { in } W_{0}^{1, \Phi}(\Omega) ; \\
u_{n} \rightarrow u_{*} \text { strongly in } L^{G}(\Omega) \text { for all N-function } G \prec \prec \Phi_{*} ; \\
u_{n} \rightarrow u_{*} \text { a.e in } \Omega
\end{array}\right.
$$

for some $u_{*} \in W_{0}^{1, \Phi}(\Omega)$.

By standard arguments, we are able to show that $J\left(u_{*}\right)=\inf _{\mathcal{N}} J$, that is,

$$
\begin{aligned}
& \int_{\Omega} \Phi\left(\left|\nabla u_{n}\right|\right) d x+\frac{1}{\delta-1} \int_{\Omega} b(x)\left|u_{n}\right|^{1-\delta} d x \stackrel{n \rightarrow \infty}{\longrightarrow} \int_{\Omega} \Phi\left(\left|\nabla u_{*}\right|\right) d x \\
& \quad+\frac{1}{\delta-1} \int_{\Omega} b(x)\left|u_{*}\right|^{1-\delta} d x
\end{aligned}
$$

holds. So, as a consequence of (32), Fatou's lemma and Lemma 2.1-(vi), we obtain

$$
\lim _{n \rightarrow \infty} \int_{\Omega} \Phi\left(\left|\nabla u_{n}\right|\right) d x=\int_{\Omega} \Phi\left(\left|\nabla u_{*}\right|\right) d x
$$


Thus, it follows from the assumption $\left(\phi_{1}\right)$, Theorem 2.4.11 and Lemma 2.4.17 in [10] that $W_{0}^{1, \Phi}(\Omega)$ is uniformly convex. This together with the weak convergence and (33), lead us to conclude that $u_{n} \rightarrow u_{*}$ in $W_{0}^{1, \Phi}(\Omega)$. After this strong convergence, we are able to follow similar arguments as done in Yijing [31] in the homogeneous case to prove that

$$
\int_{\Omega} a\left(\left|\nabla u_{*}\right|\right) \nabla u_{*} \nabla \varphi d x \geq \int_{\Omega} b(x) u_{*}^{-\delta} \varphi d x
$$

holds for any $0 \leq \varphi \in W_{0}^{1, \Phi}(\Omega)$ given. Hence, it follows from the same arguments as used to prove Lemma 3.1 that $u_{*}$ is a $W_{0}^{1, \Phi}(\Omega)$-solution of $(S)$ such that $u_{*} \geq C d$ for some $C>0$ independent of $u$.

Proof of $($ ii $) \Longrightarrow($ iii $)$. By Corollary 2.1, there exist three critical points to functional $I$, being two of them local minima and the other one a mountain pass point to energy functional $I$. So, by Theorem 1.1 we know that each one of these critical point is a solution for the problem $\left(Q_{\lambda, \mu}\right)$ that satisfy the qualitative properties claimed.

Proof of $($ iii $) \Longrightarrow(i)$. Let $0<u_{0} \in W_{0}^{1, \Phi}(\Omega)$ be a solution of $\left(Q_{\lambda, \mu}\right)$. Then $u_{0} \in$ $\operatorname{Dom}\left(\Psi_{2}\right)$, that is, $\int_{\Omega} b u_{0}^{1-\delta} d x<\infty$. These ends the proof of the equivalences.

Below, let us prove the items $(i v)$ and $(v)$. We are going to prove $(i v)$ first. Let $u=u_{a}$ be a solution of problem $\left(Q_{\lambda, \mu}\right)$. Assume by contradiction that $u \leq a$ a.e. in $\Omega$ for any $a>0$. So, it follows from $f(x, t)=f(x)$ for all $0<t<1$ and a.e. $x \in \Omega$ that $u_{a} \in W_{0}^{1, \Phi}(\Omega)$ is a solution of

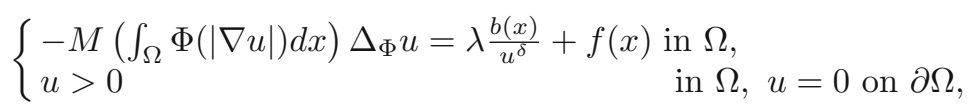

that is, $u_{a}$ is constant in $a>0$ by Lemma 4.3 .

On the other hand, by taking $\beta>\delta>1$, we have that $u_{a}^{\beta}>0$ can be used as a test function in $\left(Q_{\lambda, \mu}\right)$ and this yields the inequality

$$
\begin{aligned}
\beta M\left(\int_{\Omega} \Phi\left(\left|\nabla u_{a}\right|\right) d x\right) \int_{\Omega} a\left(\left|\nabla u_{a}\right|\right)\left|\nabla u_{a}\right| u_{a}^{\beta-1} d x & =\int_{\Omega} b u_{a}^{\beta-\delta} d x+\int_{\Omega} f\left(x, u_{a}\right) u_{a}^{\beta} \\
& \leq|b|_{1} a^{\beta-\delta}+C|\Omega|\left(1+\widetilde{H}^{-1} \circ H(a)\right) a^{\beta}
\end{aligned}
$$

for any $a>0$ given.

So, by doing $a>0$ small enough we get an absurd, because the first term of the above inequality is a positive number that does not depends on $a>0$. This ends the proof of this item.

Finally, we are going to prove $v)$. Let $u_{a}$ be a solution of problem $\left(Q_{\lambda, \mu}\right)$. Assume by contradiction that $u \leq a$ a.e. in $\Omega$ for any $a>0$ again. So, it follows that $u_{a}$ is a super solution to problem 


$$
\begin{cases}-M\left(\int_{\Omega} \Phi(|\nabla u|) d x\right) \Delta_{\Phi} u=\lambda b(x) & \text { in } \Omega, \\ u>0 & \text { in } \Omega, u=0 \text { on } \partial \Omega,\end{cases}
$$

whenever $a<1$.

On the other hand, we are able to show that the associated-energy functional to Problem (34) is coercive due the assumption $\ell \alpha>1$. So, by following standard arguments, we show that there exists a non-trivial $0 \leq v \in W_{0}^{1, \Phi}(\Omega)$ solution for the problem (34). That is, we have

$$
\begin{cases}-M\left(\int_{\Omega} \Phi\left(\left|\nabla u_{a}\right|\right) d x\right) \Delta_{\Phi} u_{a} \geq-M\left(\int_{\Omega} \Phi(|\nabla v|) d x\right) \Delta_{\Phi} v & \text { in } \Omega \\ u=v=0 & \text { on } \partial \Omega .\end{cases}
$$

So, it follows from the hypotheses that $M$ is such that a Comparison Principle holds, that $u_{a} \geq u>0$ for all $0<a \leq 1$. This fact together with the contradiction assumption lead us to have $0 \leq u \leq u_{a} \leq a$ for all $0<a \leq 1$, which is impossible for $a>0$ small enough, because $u$ is non-trivial. This ends the proof of item $v$ ) and the proof of Theorem 1.2.

Proof of Corollary 1.1. By the implication $(i \Longrightarrow i i)$ in Theorem 1.2, it suffices to exhibit a $u_{0} \in W_{0}^{1, \Phi}(\Omega)$ such that $\int_{\Omega} b u_{0}^{1-\delta} d x<\infty$. Let us construct a such one. First, we note that the regularity of the domain $\Omega$ implies that there exists an $\epsilon>0$ sufficiently small such that $d \in C^{2}\left(\bar{\Omega}_{2 \epsilon}\right)$ and $|\nabla d(x)|=1$ in $\Omega_{2 \epsilon}$, where $d(x):=\operatorname{dist}(x, \partial \Omega)$ and $\Omega_{2 \epsilon}=\{x \in \Omega: d(x)<2 \epsilon\}$. With these, define

$$
u_{0}(x)= \begin{cases}d(x)^{\theta} & \text { if } d(x)<\epsilon, \\ \epsilon^{\theta}+\int_{\epsilon}^{d(x)} \theta \epsilon^{\theta-1}\left(\frac{2 \epsilon-t}{\epsilon}\right)^{2 /\left(\phi_{-}-1\right)} d t & \text { if } \epsilon \leq d(x)<2 \epsilon, \\ \epsilon^{\theta}+\int_{\epsilon}^{2 \epsilon} \theta \epsilon^{\theta-1}\left(\frac{2 \epsilon-t}{\epsilon}\right)^{2 /\left(\phi_{-}-1\right)} d t & \text { if } \epsilon \leq d(x)<2 \epsilon\end{cases}
$$

for each $\epsilon>0$ given, where $0<\theta<1$ will be chosen later.

A simple calculation yields

$$
\nabla u_{0}(x)= \begin{cases}\theta d(x)^{\theta-1} \nabla d(x) & \text { if } d(x)<\epsilon, \\ \theta \epsilon^{\theta-1}\left(\frac{2 \epsilon-d(x)}{\epsilon}\right)^{2 /\left(\phi_{-}-1\right)} \nabla d(x) & \text { if } \epsilon \leq d(x)<2 \epsilon, \\ 0 & \text { if } \epsilon \leq d(x)<2 \epsilon\end{cases}
$$

which implies that $u_{0} \in W_{0}^{1, \Phi}(\Omega)$ if

$$
\int_{\Omega_{\epsilon}} \Phi\left(\theta d(x)^{\theta-1}|\nabla d(x)|\right) d x<\infty .
$$

Since $|\nabla d|=1$ in $\Omega_{\epsilon}$, we obtain from Lemma 5.1 in (8) that

$$
\int_{\Omega_{\epsilon}} \Phi\left(\theta d(x)^{\theta-1}|\nabla d(x)|\right) d x=\int_{\Omega_{\epsilon}} \Phi\left(\theta d(x)^{\theta-1}\right) d x \stackrel{\theta<1}{\leq} C \int_{\Omega_{\epsilon}} d(x)^{(\theta-1) \phi_{+}} d x
$$

that lead us to show (35) for $\theta$ such that $(\theta-1) \phi_{+}>-1$, due well-known 
result in [19]. That is, for $\operatorname{such} \theta$, we have that $u_{0} \in W_{0}^{1, \Phi}(\Omega)$.

To complete the exhibition, if $0<\theta<1$ is such that $\theta q(1-\delta)>1-q$, we have

$$
\int_{\Omega_{\epsilon}} b(x) d(x)^{\theta(1-\delta)} d x \leq\left(\int_{\Omega} b(x)^{q} d x\right)^{1 / q}\left(\int_{\Omega_{\epsilon}} d(x)^{\theta(1-\delta) q^{\prime}} d x<\infty\right)^{1 / q^{\prime}}<\infty,
$$

because $b \in L^{q}(\Omega)$ and the result in [19] again.

Finally, to occur (35) and (4) simultaneously, we have to be able to choose a $0<\theta<1$ satisfying at same time $(\theta-1) \phi_{+}>-1$ and $\theta q(1-\delta)>1-q$. We can do these by controlling the range of $\delta$. Since

$$
1-\frac{1}{\phi_{+}}<\frac{q-1}{q(\delta-1)} \text { if, and only if, } 0<\delta<\frac{q\left(2 \phi_{+}-1\right)-\phi_{+}}{q\left(\phi_{+}-1\right)},
$$

we are able to pick a

$$
\theta \in\left(1-\frac{1}{\phi_{+}}, \min \left\{1, \frac{q-1}{q(\delta-1)}\right\}\right) \subset(0,1),
$$

whenever $\delta$ range as above. This proves that $u_{0}$, defined as above, satisfies the condition of item $(i)$ in Theorem 1.2. This finishes the proof.

\section{Acknowledgements}

The research of Vicenţiu D. Rădulescu was supported by a Grant of the Ministry of Research, Innovation and Digitization, CNCS/CCCDI-UEFISCDI, Project No. PCE 137/2021, within PNCDI III. Carlos Alberto Santos acknowledges the support of CNPq/Brazil Proc. No 311562/2020-5.

Open Access. This article is licensed under a Creative Commons Attribution 4.0 International License, which permits use, sharing, adaptation, distribution and reproduction in any medium or format, as long as you give appropriate credit to the original author(s) and the source, provide a link to the Creative Commons licence, and indicate if changes were made. The images or other third party material in this article are included in the article's Creative Commons licence, unless indicated otherwise in a credit line to the material. If material is not included in the article's Creative Commons licence and your intended use is not permitted by statutory regulation or exceeds the permitted use, you will need to obtain permission directly from the copyright holder. To view a copy of this licence, visit http://creativecommons. org/licenses/by/4.0/.

Publisher's Note Springer Nature remains neutral with regard to jurisdictional claims in published maps and institutional affiliations.

\section{References}

[1] Adams, R.A.: Sobolev Spaces, Pure and Applied Mathematics, Vol. 65. Academic Press [A subsidiary of Harcourt Brace Jovanovich, Publishers], New YorkLondon (1975) 
[2] Ambrosetti, A., Arcoya, D.: Remarks on non homogeneous elliptic Kirchhoff equations. Nonlinear Differ. Equ. Appl. 23, 57 (2016)

[3] Cammaroto, F., Vilasi, L.: Multiple solutions for a Kirchhoff-type problem involving the $p(x)$-Laplacian operator. Nonlinear Anal. Theory Methods Appl. 74(5), 1841-1852 (2011)

[4] Carl, S., Le, V.K., Motreanu, D.: Nonsmooth Variational Problems and Their Inequalities. Comparison Principles and Applications. Springer Monographs in Mathematics. Springer, New York (2007)

[5] Chang, K.C.: Variational methods for nondifferentiable functionals and their applications to partial differential equations. J. Math. Anal. Appl. 80(1), 102$129(1981)$

[6] Cianchi, A., Maz'ya, V.: Second-order two-sided estimates in nonlinear elliptic problems. Arch. Rational Mech. Anal. 229, 569-599 (2018)

[7] Cianchi, A., Maz'ya, V.: Optimal second-order regularity for the $p$-Laplace system. J. Math. Pures Appl. 132, 41-78 (2019)

[8] Clarke, F.H.: Optimization and Nonsmooth Analysis. SIAM, Philadelphia (1990)

[9] Corrêa, F.J.S.A., Gonçalves, J.V.: Sublinear elliptic systems with discontinuous nonlinearities. Appl. Anal. 44, 37-50 (1990)

[10] Diening, L., Harjulehto, P., Hästö, P., Ružička, M.: Lebesgue and Sobolev Spaces with Variable Exponents. Lecture Notes in Mathematics, Springer, Heidelberg (2011)

[11] Fang, F., Tan, Z.: Existence of three solutions for quasilinear elliptic equations: an Orlicz-Sobolev space setting. Acta Math. Appl. Sin. Engl. Ser. 33(2), 287-296 (2017)

[12] Faraci, F., Smyrlis, G.: Three solutions for a class of higher dimensional singular problems. NoDEA Nonlinear Differ. Equ. Appl. 23(14), 23-45 (2016)

[13] Faraci, F., Smyrlis, G.: Three solutions for a singular quasilinear elliptic problem. Proc. Edinb. Math. Soc. 62(1), 179-196 (2019)

[14] Gonçalves, J.V., Carvalho, M.L.M., Santos, C.A.: Quasilinear elliptic systems with convex-concave singular terms and $\Phi$-Laplacian operator. Differ. Integr. Equ. 31(2), 231-256 (2018)

[15] Gonçalves, J.V., Carvalho, M.L., Santos, C.A.: About positive $W_{l o c}^{1, \Phi}(\Omega)$ solutions to quasilinear elliptic problems with singular semilinear term. Topol. Methods Nonlinear Anal. 53(2), 491-517 (2019)

[16] Guedda, M., Véron, L.: Quasilinear elliptic equations involving critical Sobolev exponents. Nonlinear Anal. 13(8), 879-902 (1989)

[17] Krasnosels'kii, M.A., Rutickii, J.B.: Convex Functions and Orlicz Spaces. Noordhoff Ltd., Groningen (1961) 
[18] Kufner, A., John, O., Fŭcík, S.: Function spaces,. Academia Noordhoff International Publishing, Pragu, Leyden (1977)

[19] Lazer, A.C., McKenna, P.J.: On a singular nonlinear elliptic boundary-value problem. Proc. Amer. Math. Soc. 111(3), 721-730 (1991)

[20] Le, V.K., Motreanu, D., Motreanu, V.V.: On a non-smooth eigenvalue problem in Orlicz-Sobolev spaces. Appl. Anal. 89(2), 229-242 (2010)

[21] Liang, S., Pucci, P., Zhang, B.: Multiple solutions for critical ChoquardKirchhoff type equations. Adv. Nonlinear Anal. 10(1), 400-419 (2021)

[22] Lou, H.: On singular sets of local solutions to $p$-Laplace equations. Chin. Ann. Math. 29B, 521-530 (2008)

[23] Marano, S.A., Motreanu, D.: On a three critical points theorem for nondifferentiable functions and applications to nonlinear boundary value problems. Nonlinear Anal. 48, 37-52 (2002)

[24] Mingione, G., Rădulescu, V.: Recent developments in problems with nonstandard growth and nonuniform ellipticity. J. Math. Anal. Appl. 501, 125197 (2021)

[25] Mohammed, A.: Positive solutions of the $p$-Laplace equation with singular nonlinearity. J. Math. Anal. Appl. 352(1), 234-245 (2009)

[26] Mukherjee ,T., Pucci, P., Xiang, M.: Combined effects of singular and exponential nonlinearities in fractional Kirchhoff problems. Discrete Contin. Dyn. Syst.. https://doi.org/10.3934/dcds.2021111.

[27] Rao, M.M., Ren, Z.D.: Theory of Orlicz Spaces. Marcel Dekker Inc, New York (1991)

[28] Ricceri, B.: Sublevel sets and global minima of coercive functionals and local minima of their perturbations. J. Nonlinear Convex Anal. 5(2), 157-168 (2004)

[29] Ricceri, B.: A further three critical points theorem. Nonlinear Anal. 71(9), 41514157 (2009)

[30] Ricceri, B.: On an elliptic Kirchhoff-type problem depending on two parameters. J. Glob. Optim. 46(4), 543-549 (2010)

[31] Sun, Y.: Compatibility phenomena in singular problems. Proc. Roy. Soc. Edinb. Sect. A 143(6), 1321-1330 (2013)

[32] Szulkin, A.: Minimax principles for lower semicontinuous functions and applications to nonlinear boundary value problems. Ann. Inst. H. Poincaré Anal. Non Linéaire 3(2), 77-109 (1986)

[33] Zhao, L., He, Y., Zhao, P.: The existence of three positive solutions of a singular p-Laplacian problem. Nonlinear Anal. 74(16), 5745-5753 (2011) 
Vicenţiu D. Rădulescu

Faculty of Applied Mathematics

AGH University of Science and Technology

30-059 Kraków

Poland

and

Department of Mathematics

University of Craiova

200585 Craiova

Romania

and

Simion Stoilow Institute of Mathematics of the Romanian Academy

P.O. Box 1-764014700 Bucharest

Romania

e-mail: radulescu@inf.ucv.ro

Carlos Alberto Santos

Departamento de Matemática

Universidade de Brasília

70910-900 Brasília DF

Brazil

e-mail: csantos@unb.br

Lais Santos

Departamento de Matemática

Universidade Federal de Viçosa

36570-000 Viçosa MG

Brazil

e-mail: matmslais@gmail.com

Marcos L. M. Carvalho

Instituto de Matemática

Universidade Federal de Goiás

74690-900 Goiânia GO

Brazil

e-mail: marcos_leandro_carvalho@ufg.br

Received: 23 April 2021.

Accepted: 15 September 2021. 